\title{
A New Equalization Performance Analyzing Method for Blind Adaptive Equalizers Inspired by Maximum Time Interval Error
}

\author{
Guilad Suissa, Monika Pinchas \\ Department of Electrical and Electronic Engineering, Ariel University, Ariel, Israel \\ Email: guilad.suissa@gmail.com,monika.pinchas@gmail.com, monikap@ariel.ac.il
}

How to cite this paper: Suissa, G. and Pinchas, M. (2017) A New Equalization Performance Analyzing Method for Blind Adaptive Equalizers Inspired by Maximum Time Interval Error. Journal of Signal and Information Processing, 8, 42-64. https://doi.org/10.4236/jsip.2017.82004

Received: February 22, 2017

Accepted: May 7, 2017

Published: May 10, 2017

Copyright (c) 2017 by authors and Scientific Research Publishing Inc. This work is licensed under the Creative Commons Attribution International License (CC BY 4.0).

http://creativecommons.org/licenses/by/4.0/

\begin{abstract}
Up to now, the Mean Square Error (MSE) criteria, the residual Inter-Symbol Interference (ISI) and the Bit-Error-Rate (BER) were used to analyze the equalization performance of a blind adaptive equalizer in its convergence state. In this paper, we propose an additional tool (additional to the ISI, MSE and BER) for analyzing the equalization performance in the convergence region based on the Maximum Time Interval Error (MTIE) criterion that is used for the specification of clock stability requirements in telecommunications standards. This new tool preserves the short term statistical information unlike the already known tools (BER, ISI, MSE) that lack this information. Simulation results will show that the equalization performance of a blind adaptive equalizer obtained in the convergence region for two different channels is seen to be approximately the same from the residual ISI and MSE point of view while this is not the case with our new proposed tool. Thus, our new proposed tool might be considered as a more sensitive tool compared to the ISI and MSE method.
\end{abstract}

\section{Keywords}

Blind Equalizer, ISI, MSE, BER, MTIE

\section{Introduction}

In data communication, signals transmitted between remote locations often encounter a signal-altering physical channel (in wired communications or in wireless communications). These physical channels may cause signal distortion, including echoes and frequency-selective filtering of the transmitted signal [1]. In digital communications, a critical manifestation of distortion is ISI, whereby symbols transmitted before and after a given symbol corrupt the detection of 
that symbol. All physical channels (at high enough data rates) tend to exhibit ISI [1].

An effective way to overcome the ISI is using adaptive equalization technology. An adaptive equalizer is an inverse filter which reduces the effects of ISI by deconvolving the transmitted data sequence from the time varying channel response [2]. The conventional approach for adaptive filtering usually requires a training sequence where the desired response is compared to the received symbols and an estimated error is produced which helps adjusting the coefficients of the adaptive filter [3].

When a training session is impossible or very costly, blind equalizers are a convenient solution. Blind equalization algorithms are essentially adaptive filtering algorithms designed such that they do not require the external supply of a desired response to generate the error signal in the output of the adaptive equalization filter [4]. The algorithm itself generates an estimate of the desired response by applying a nonlinear transformation to sequences involved in the adaptation process [4]. Adaptive equalizers are widely used in digital communications systems to remove the ISI introduced by dispersive channels [5]. In order to avoid the transmission of pilot sequences and use the channel bandwidth in an efficient manner the blind equalization techniques are highly desirable. A popular approach for the blind adaptation of finite impulse response (FIR) equalizers is the constant modulus algorithm [6] [7] and its variant known as multi modulus algorithm [8] [9] [10] due to their low computational cost [5].

Today, in order to analyze the equalization performance, namely, to see how much the equalizer overcomes the ISI, the ISI, the MSE or the BER are simulated. The ISI, BER and MSE provide long term statistical information in the steady state region (convergence state). Thus, for instance, there may be cases where two different simulation results obtained in the convergence region with different channels (but using the same algorithm for reducing the ISI), may lead approximately to the same residual ISI but may have different short term statistical information. Namely, in the short term, there may be seen different amounts of errors for the two different channels. Thus, one channel is preferable over the other. Therefore, the following question may arise: is it possible to get also short term statistical information of the blind adaptive equalization performance in the convergence region?

A major topic of discussion in standard bodies dealing with network synchronization [11] [12] [13] is clock noise characterization and measurement [14]. MTIE is historically one of the main time-domain quantities for the specification of clock stability requirements in telecommunications standards [14]. Among the quantities considered in international standards for specification of phase and frequency stability requirements, the MTIE has played historically a major role for characterizing time and frequency performance in digital telecommunications networks [15] [16] [17] [18] [19] and is a rough measure of the peak time deviation of a clock with respect to a known reference [20].

The purpose of this work is to provide an additional tool (additional to the ISI, 
MSE and BER) for diagnosing equalization performance in the steady state region based on the MTIE method used in the telecommunication area. Simulation results will show that our new proposed tool provides us short term as well as long term statistical information and is able to show differences in the equalization performance comparison obtained in the convergence state even when it is quite difficult to see it with the MSE and ISI method.

The paper is organized as follows: after having described the system under consideration in Section II, Section III describes our new proposed tool for analyzing the equalization performance in the convergence region based on the MTIE. In Section IV simulation results are given using our new proposed tool compared with the existing methods (MSE, ISI) and Section V is our conclusion.

\section{System Description}

In this section we consider the system described in Figure 1 with the following assumptions:

1) The input sequence $x[n]$ belongs to a real or two independent quadrature carrier case constellation input with variance $\sigma_{x}^{2}$ where $x_{1}[n]$ and $x_{2}[n]$ are the real and imaginary parts of $x[n]$ respectively.

2) The unknown channel $h[n]$ is a possibly non-minimum phase linear time-invariant filter, FIR filter.

3) The equalizer $c[n]$ is a FIR filter.

4) The noise $w[n]$ is an additive Gaussian white noise with zero mean and variance $\sigma_{w}^{2}=E\left[w[n] w^{*}[n]\right]\left(E[\cdot]\right.$ is the expectation operator and ()$^{*}$ is the conjugate operation).

For simplicity, we use in this paper only the 16QAM constellation input (Figure 2) for $x[n]$. The sequence $x[n]$ is transmitted through the channel $h[n]$ and is corrupted with noise $w[n]$. Therefore, the equalizer's input sequence $y[n]$ may be written as:

$$
y[n]=x[n] * h[n]+w[n]
$$

where $" * "$ denotes the convolution operation. The equalized output sequence is defined by:

$$
z[n]=y[n] * c[n]=x[n] * h[n] * c[n]+w[n] * c[n]=x[n]+e[n]
$$

where $e[n]$ is the sum of the convolutional error due to non-ideal coefficients

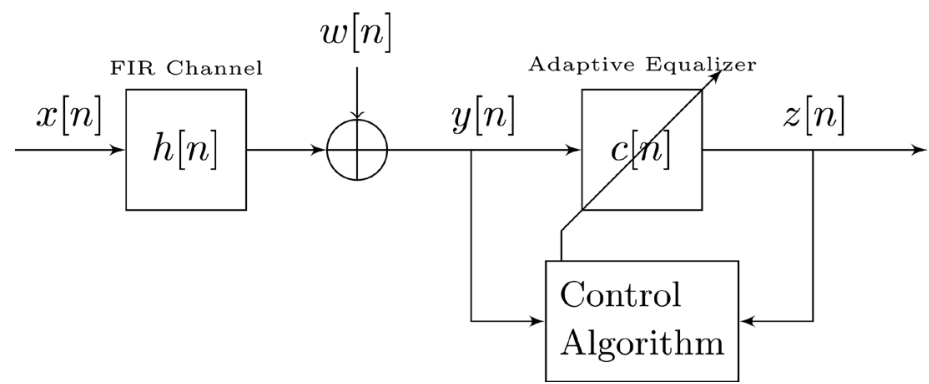

Figure 1. Block diagram of a communication system. 


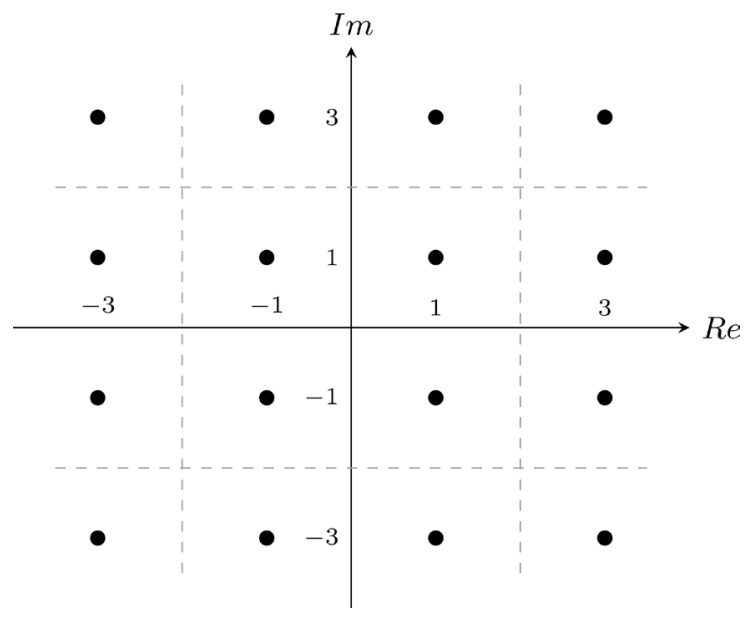

Figure 2. 16QAM Constellation Diagram.

of the equalizer $\left(c[n]^{*} h[n] \neq \delta[n]\right.$ where $\delta$ is the Kronecker delta function) and the noise error passed via the filter (equalizer). It should be pointed out that for the noiseless and ideal case the equalized output is a delayed version of the input multiplied by a constant phase shift $\left(z[n]=x[n-D] e^{j \theta}\right.$ where $D$ is a constant delay and $\theta$ is a constant phase shift). But, according to [21], we can assume in (2) that $D=0$ and $\theta=0$, since $D$ does not affect the reconstruction of the original input sequence $x[n]$ and $\theta$ can be removed by a decision device. Next we turn to the adaptation mechanism of the equalizer [22]-[28] by using Godard's algorithm [6]:

$$
c_{m}[n+1]=c_{m}[n]-\mu_{G}\left(|z[n]|^{2}-\frac{E\left[|x[n]|^{4}\right]}{E\left[|x[n]|^{2}\right]}\right) z[n] y^{*}[n-m]
$$

where $\mu_{G}$ is the step-size parameter, $m=0,1,2,3, \cdots, N-1$ and $N$ is the equalizer's tap length.

\section{New Tool for Equalization Performance Analysis}

In this section we introduce our new proposed tool for the blind equalization performance analysis based on a network clock synchronization measurement method, namely, the MTIE measurement method.

For a given clock, the time error function $T E(t)$ between its time $T(t)$ and a reference time $T_{\text {ref }}(t)$ is defined as [14] [20] [29]:

$$
T E(t)=T(t)-T_{r e f}(t)
$$

Thus, the $\operatorname{MTIE}(\tau, T)$ which is the maximum peak-to-peak variation of $T E(t)$ (4) for all the possible observation intervals $\tau$ within a measurement period $T$ (see Figure 3 recalled from [20]) can be defined according to [14] [20] [29] as:

$$
\operatorname{MTIE}(\tau, T)=\max _{0 \leq t_{0} \leq T-\tau}\left\{\max _{t_{0} \leq t \leq t_{0}+\tau}[T E(t)]-\min _{t_{0} \leq t \leq t_{0}+\tau}[T E(t)]\right\}
$$




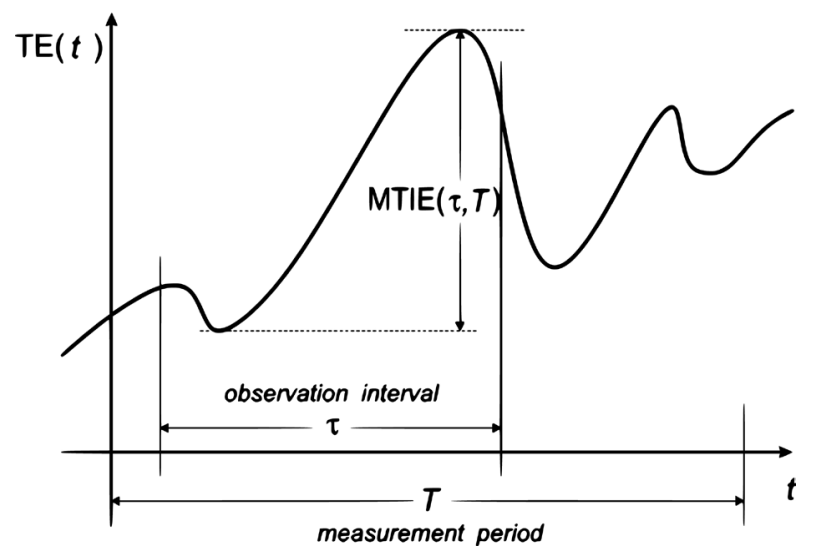

Figure 3. Definition of $\operatorname{MTIE}(\tau, T)$.

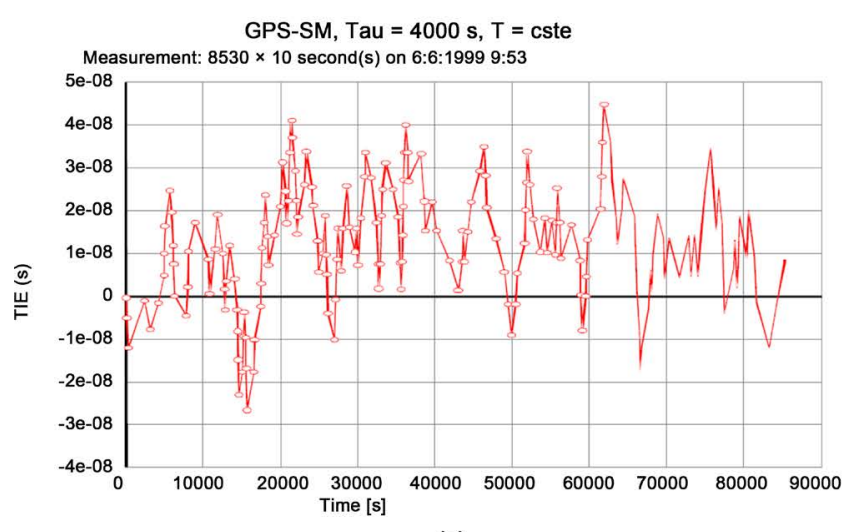

(a)

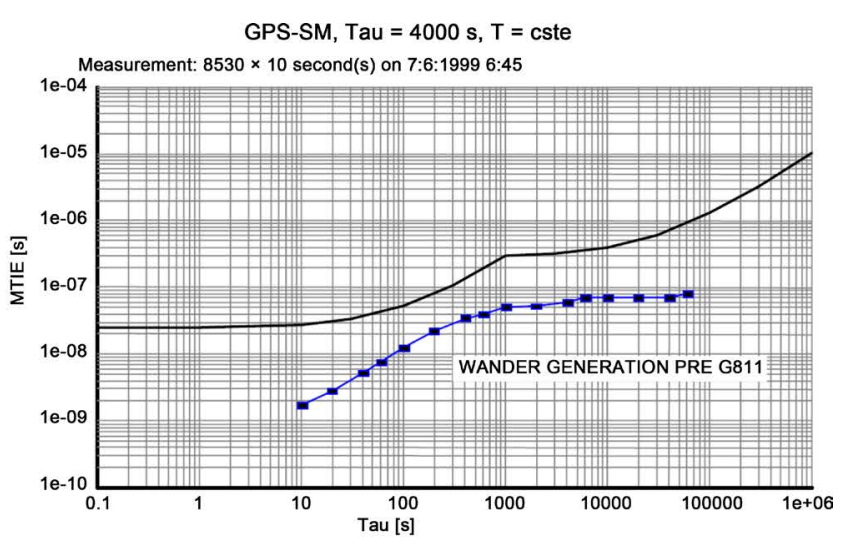

(b)

Figure 4. The clock TIE and MTIE measurements in the OSA 4520 GPS-SP, a stand-alone GPS receiver. (a) The clock TIE measurement the in OSA 4520 GPS-SP GPS receiver. (b) The lower line is the clock MTIE measurement in the OSA 4520 GPS-SP GPS receiver while the upper line is ITU-T G.811 recommendation.

An example of a MTIE measurement of a clock is shown in Figure 4 (recalled from [30]) where Figure 4(a) and Figure 4(b) are the TIE and MTIE measurements respectively according to [20]. Please note that according to Figure 4(b) the clock has less time errors for small intervals while the time error increases for bigger intervals i.e. the clock has less time errors in short term and the time error increases in long term.

Next, we adopt the concept of the MTIE measurement method from the telecommunication area to the world of equalization performance. Based on (2), we denote

$$
\operatorname{ConE}[n] \equiv e[n]=z[n]-x[n]
$$

Since $x[n]$ belongs to a real or two independent quadrature carrier case constellation input, we refer in the following only to the real parts of $z[n]$ and $x[n]$ to produce $\operatorname{ConE}[n]$ (6). Next, based on (5), we introduce $M \operatorname{ConE}\left(\tau^{\prime}\right)$, the maximum peak-to-peak variation of $\operatorname{Con} E[n]$ (6) for all the possible observation intervals $\tau^{\prime}$ (see Figure 5) which can be defined as:

$$
\operatorname{MConE}\left(\tau^{\prime}\right)=\max _{1 \leq k \leq N-\tau^{\prime}}\left\{\max _{k \leq n \leq k+\tau^{\prime}} e[n]-\min _{k \leq n \leq k+\tau^{\prime}} e[n]\right\}
$$




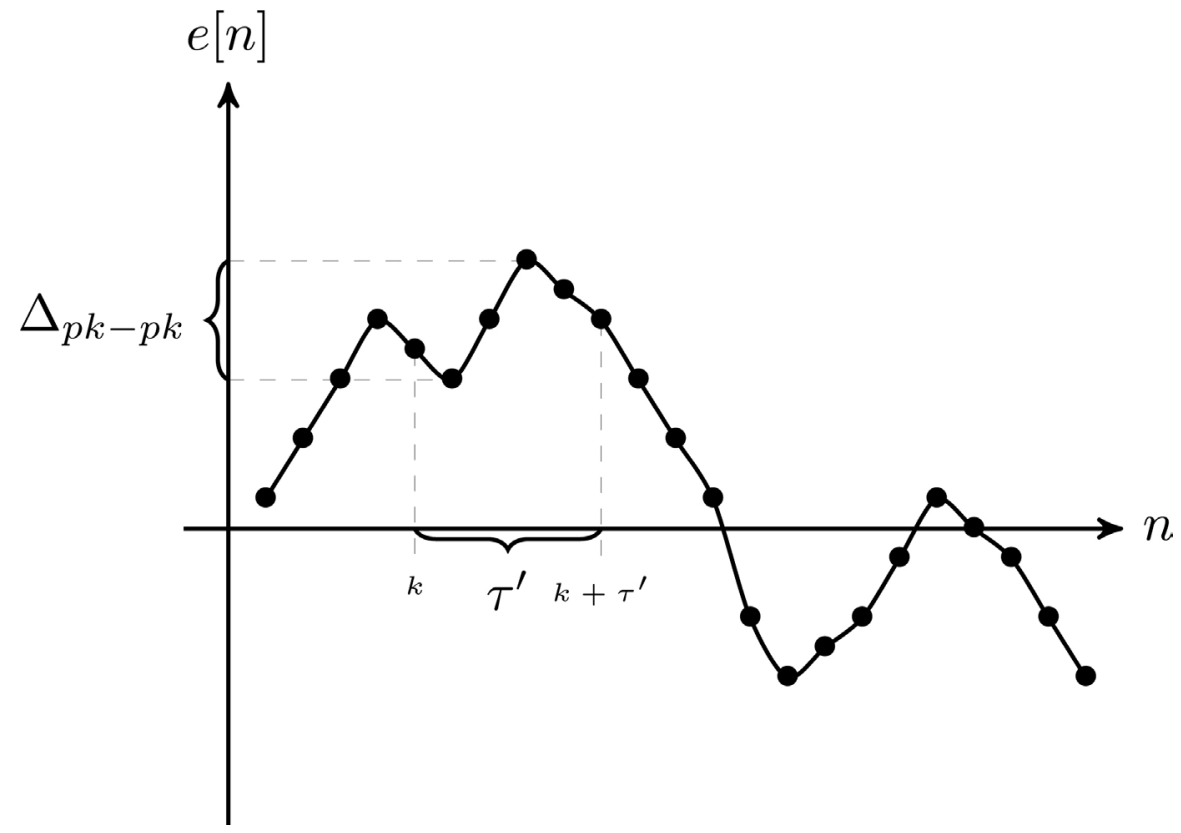

Figure 5. Peak-to-peak of the error sequence $e[n]$ within $\tau^{\prime}$ samples.

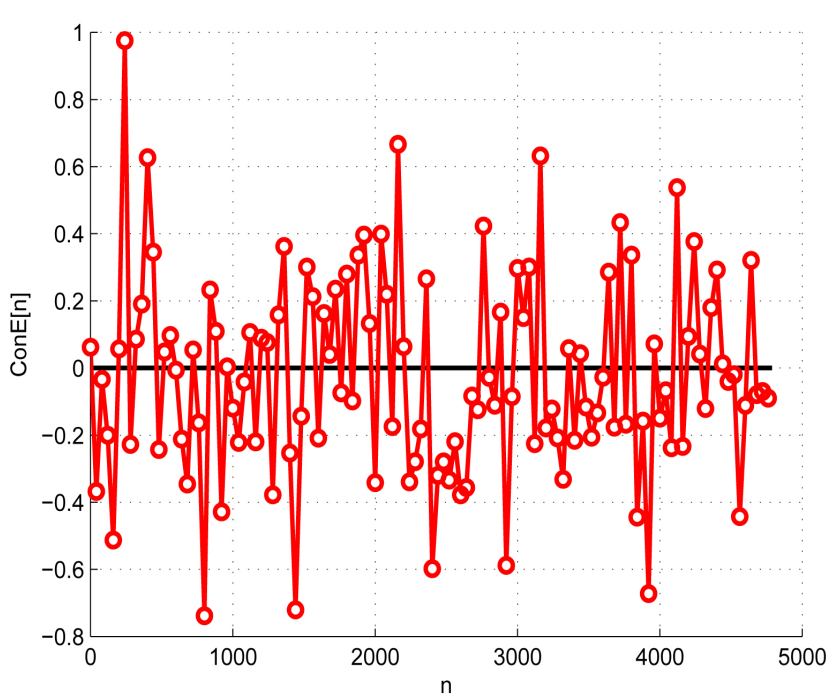

(a)

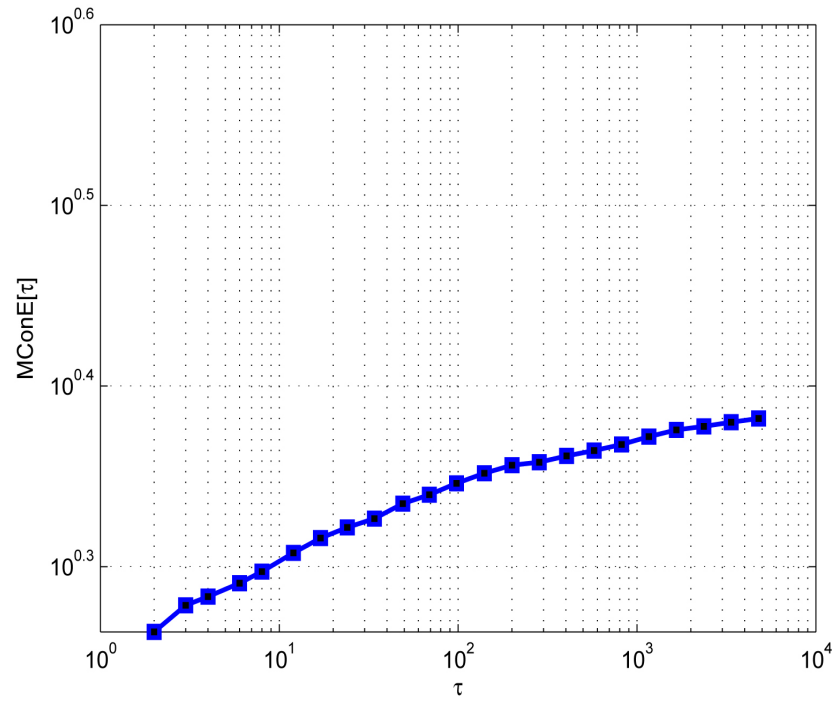

(b)

Figure 6. ConE and MConE measurements of an equalization process using Godard's algorithm with Signal to Noise Ratio (SNR) $20[\mathrm{~dB}]$, the equalizer step size was set to $3 \times 10^{-5}$. The equalizer's tap length was set to 13 . (a) ConE measurement of an equalization process. (b) MConE measurement of an equalization process.

where $\tau^{\prime}$ is the length of the interval window (in terms of discrete samples).

An example for a MConE measurement belonging to an equalization process in the convergence state is shown in Figure 6 where Figure 6(a) and Figure 6(b) are the ConE (6) and MConE (7) measurements respectively.

The resemblance between the example in Figure 4 and the example in Figure 6 is due to the nature of the time error (Figure $4(a)$ ) and the error $e[n]$ (Figure 6(a)). In both TE (4) and ConE (6) calculations, a reference signal is needed $\left(T_{r e f}(t)\right.$ and $x[n]$ for the TE and ConE calculations respectively). 


\section{Simulations Results}

In this section we present several simulation results using the MConE tool for obtaining the blind adaptive equalization performance using Godard's algorithm [6] with a 16QAM input sequence for $x[n]$, compared to the existing methods (ISI and MSE).

As already mentioned earlier in this paper, our new proposed tool for diagnosing equalization performance in the steady state region might be considered as a more sensitive tool compared to the ISI and MSE method. But, it should be kept in mind that not every difference seen in the equalization performance comparison with our new proposed tool automatically leads to errors in the recovered symbols. Thus, to see this, we denote in the following Error Accumulation as $E_{-} A[n]$ (8) defined by:

$$
E_{-} A[n]= \begin{cases}E_{-} A[n-1]+1, & \text { if }|e[n]| \geq \varepsilon \\ E_{-} A[n-1] & \text { otherwise }\end{cases}
$$

where $E_{-} A[0]=0$ and $\varepsilon$ is the distinction threshold (e.g. in 16QAM the threshold is \pm 1 for the real or the imaginary part). Since we deal in this paper with real or two independent quadrature carrier case constellation (16 QAM), we consider only the real parts of $z[n]$ and $x[n]$ for calculating (6), (7) and (8). From (8), the probability of error as a function of time can be obtained. The following channels $h[n]$ were used:

Channel 1 according to [31]:

$$
\begin{aligned}
h[n]= & (-0.0144,0.0006,0.0427,0.0090,-0.4842, \\
& -0.0376,0.8163,0.0247,0.2976,0.0122,0.0764, \\
& 0.0111,0.0162,0.0063) .
\end{aligned}
$$

Channel 2 according to [22]:

$$
\begin{aligned}
h[n]= & (-0.0144,0.0006,0.03427,-0.3090,-0.03842, \\
& 0.8376,0.04163,0.4247,0.02976,0.08122,0.04764, \\
& 0.0411,0.0162,0.0063) .
\end{aligned}
$$

Channel 3 according to [32]:

$$
h[n]= \begin{cases}0, & \text { for } n<0 \\ -0.4, & \text { for } n=0 \\ 0.840 .4^{n-1}, & \text { for } n>0\end{cases}
$$

Figures 7-10 show the simulation results for the ISI, MSE, MConE and Accumulated Error (8) respectively for various tap length values $(N=11,13,15)$, $\mathrm{SNR}=20[\mathrm{~dB}]$ and $\mu_{G}=3 \times 10^{-5}$. According to Figure 8 it is very difficult to see for which equalizer's tap length $(N)$, better equalization performance is obtained from the MSE point of view. From Figure 7 the equalization performance from the ISI point of view is very close for the case of $N=13$ and $N=15$. However, according to Figure 9, the difference in the equalization performance between the various equalizer's tap length is seen very clearly. The difference in the equalization performance for the various equalizer's tap length is also seen in 
Figure 10 in the short range while in the long term the difference in the equalization performance resembles the difference in equalization performance as is seen in Figure 7.

Figures 11-14 show the simulation results for the ISI, MSE, MConE and Accumulated Error (8) respectively for two different channels (channel $2(\mathrm{CH} 2)$ and channel $3(\mathrm{CH} 3))$ and for two different step sizes $\left(\mu_{G}=4 \times 10^{-5}\right.$ for channel

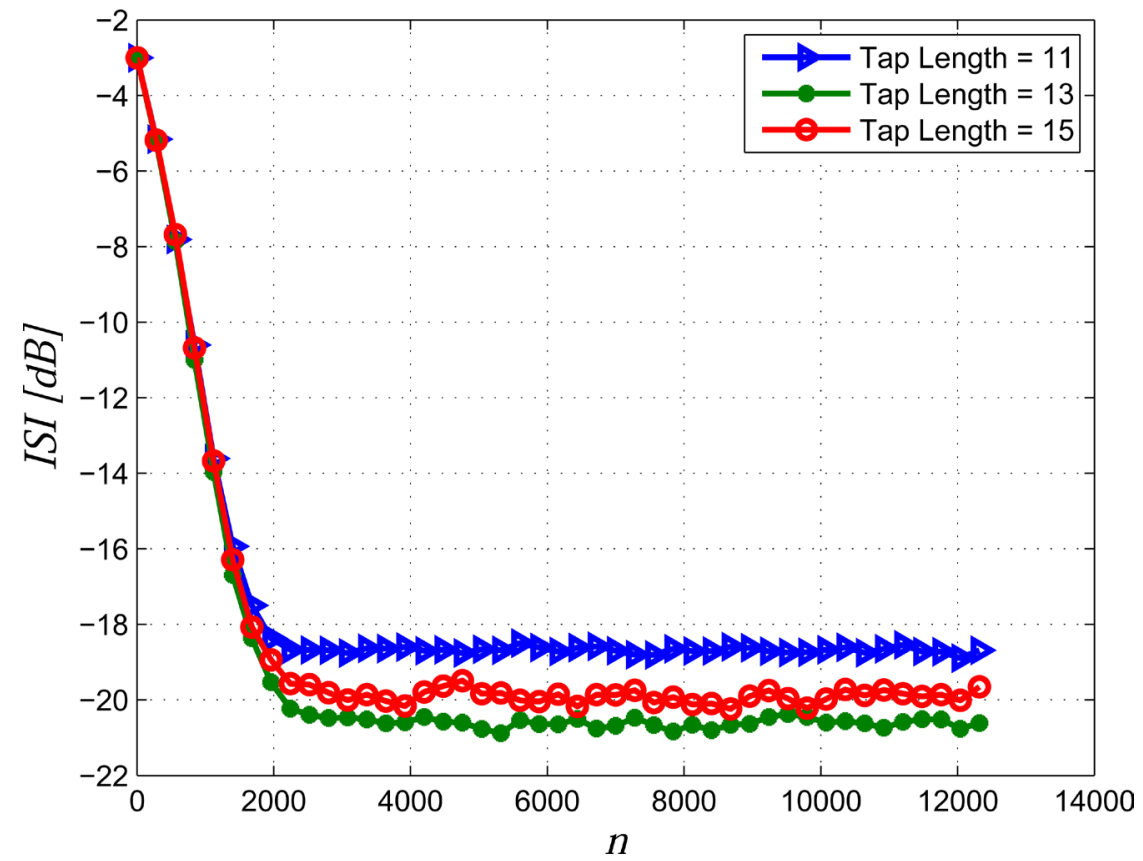

Figure 7. ISI as a function of iteration number for various equalizer's tap length. The averaged results were obtained from 50 Monte Carlo trials.

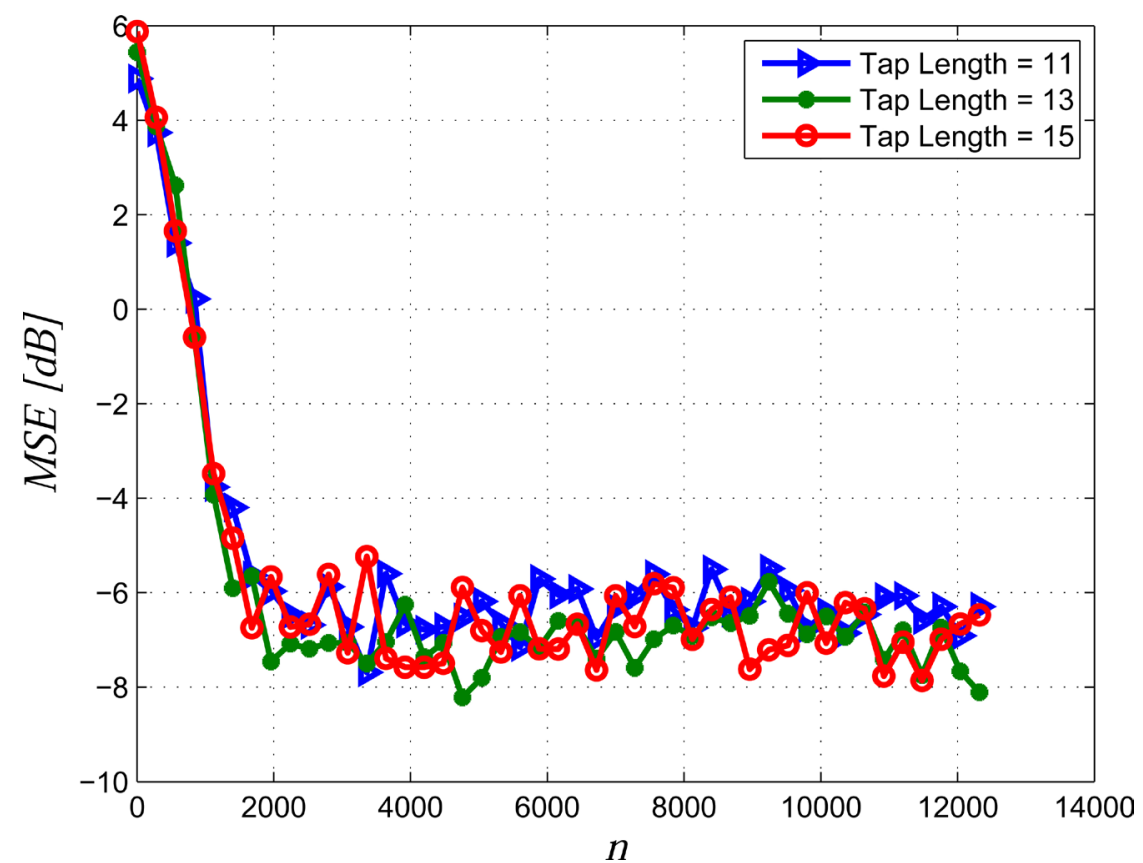

Figure 8. MSE as a function of iteration number for various equalizer's tap length. The averaged results were obtained from 50 Monte Carlo trials. 


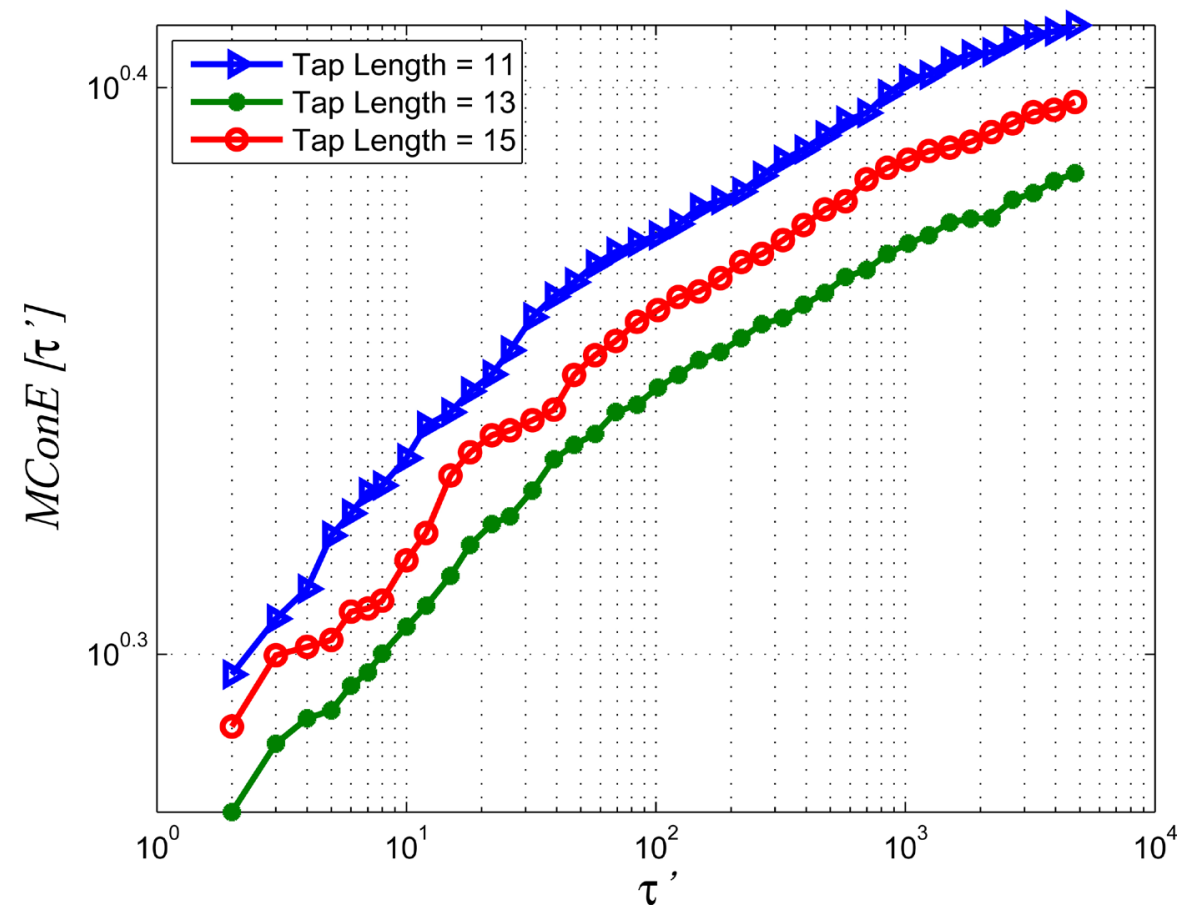

Figure 9. MConE as a function of the window length for various equalizer's tap length. The averaged results were obtained from 50 Monte Carlo trials.

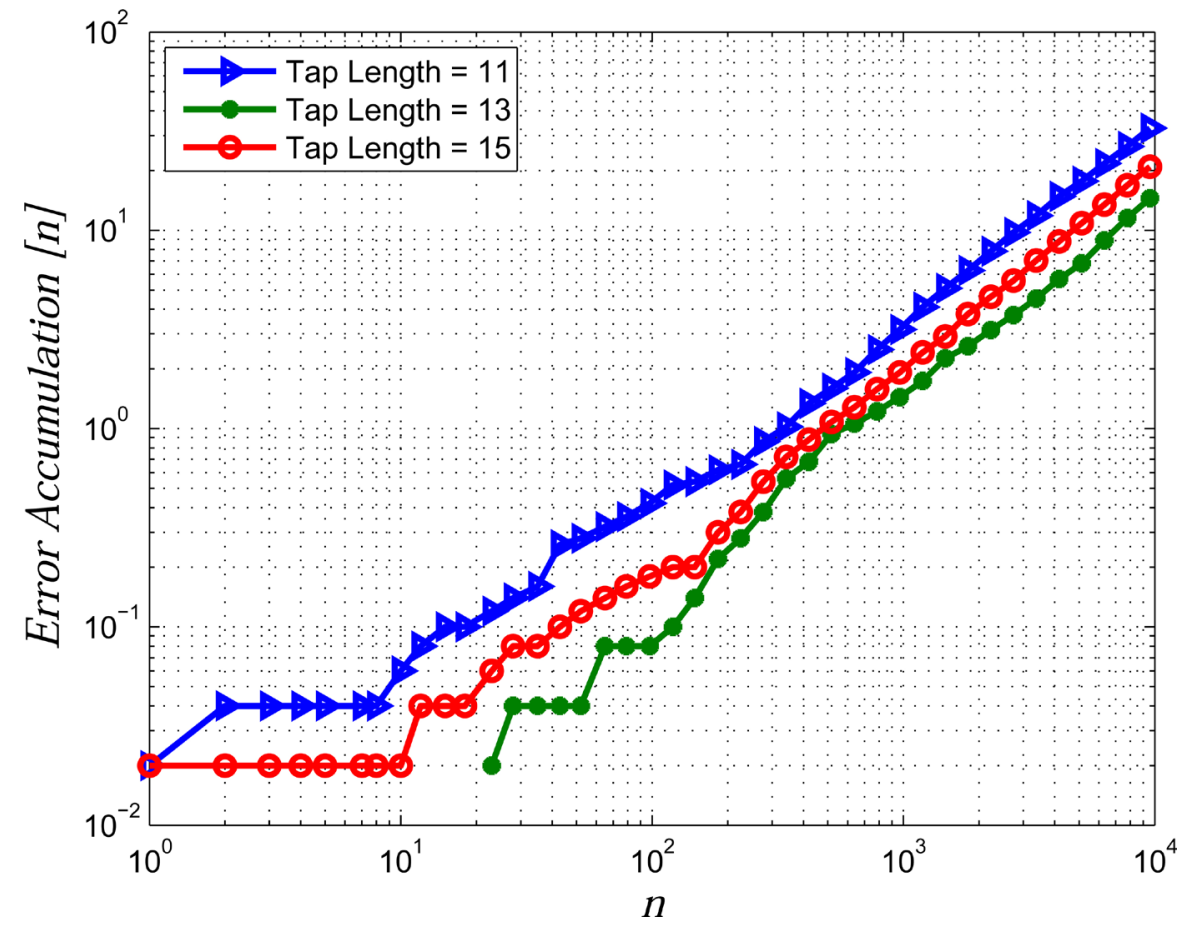

Figure 10. Error Accumulation as a function of iteration number for various equalizer's tap length. The averaged results were obtained from 50 Monte Carlo trials. 


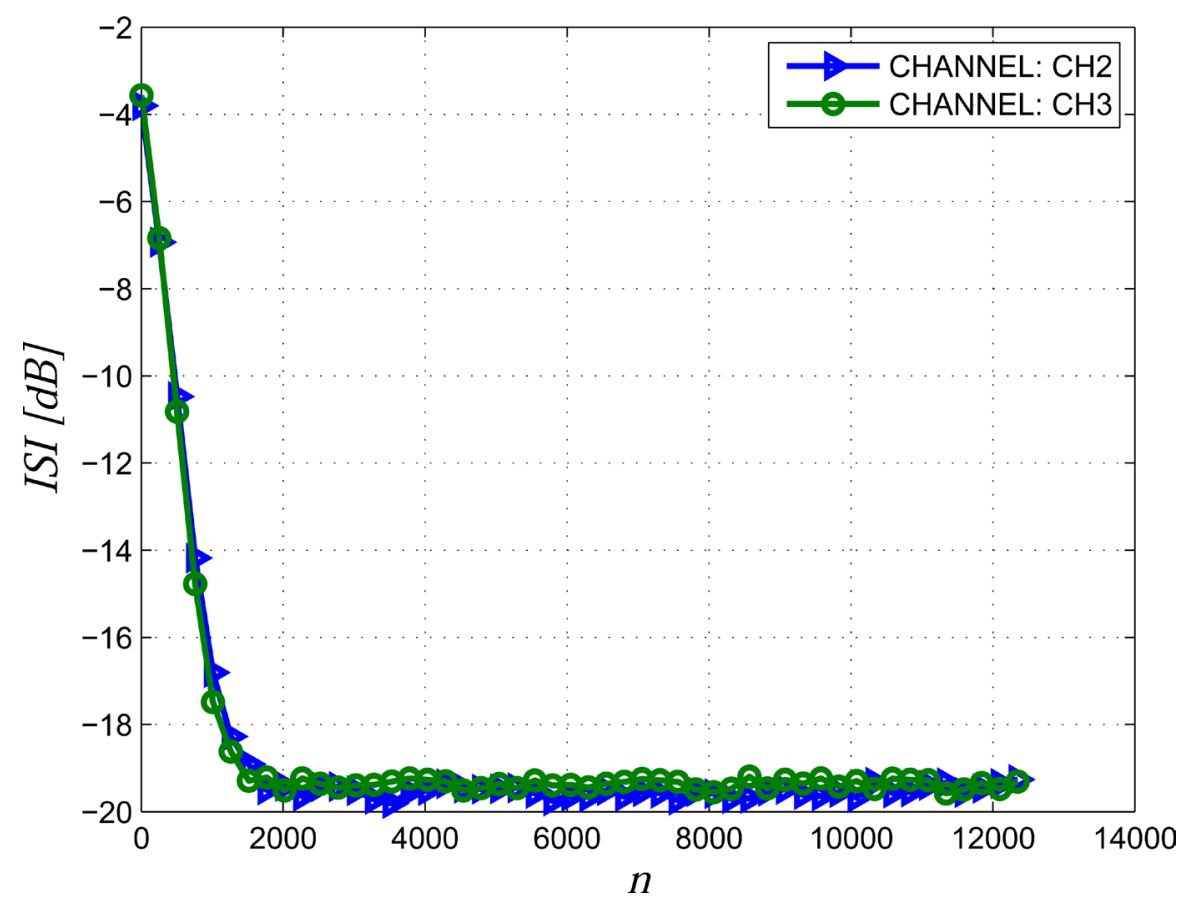

Figure 11. ISI as a function of iteration number for two channel cases. The averaged results were obtained from 100 Monte Carlo trials.

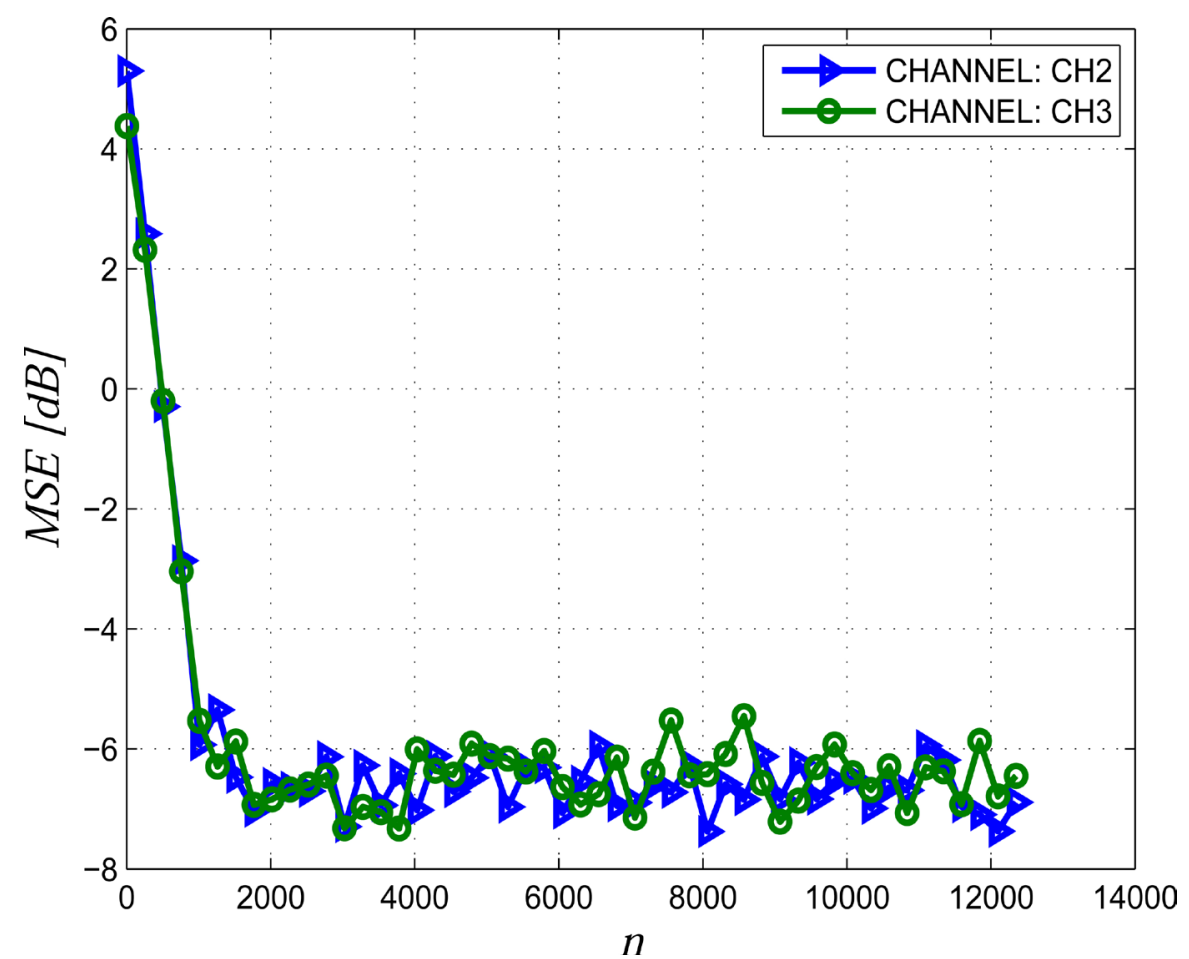

Figure 12. MSE as a function of iteration number for two channel cases. The averaged results were obtained from 100 Monte Carlo trials. 


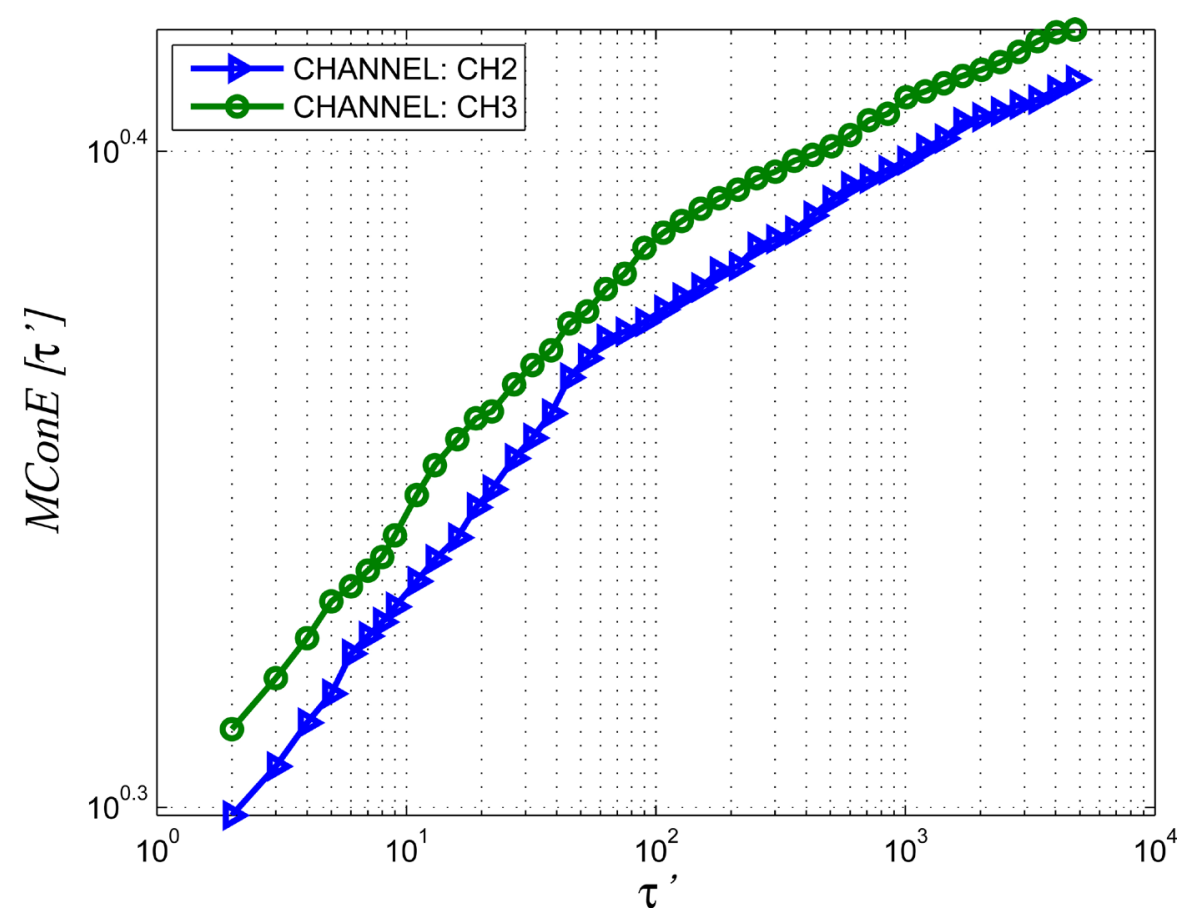

Figure 13. MConE as a function of the window length for two channel cases. The averaged results were obtained from 100 Monte Carlo trials.

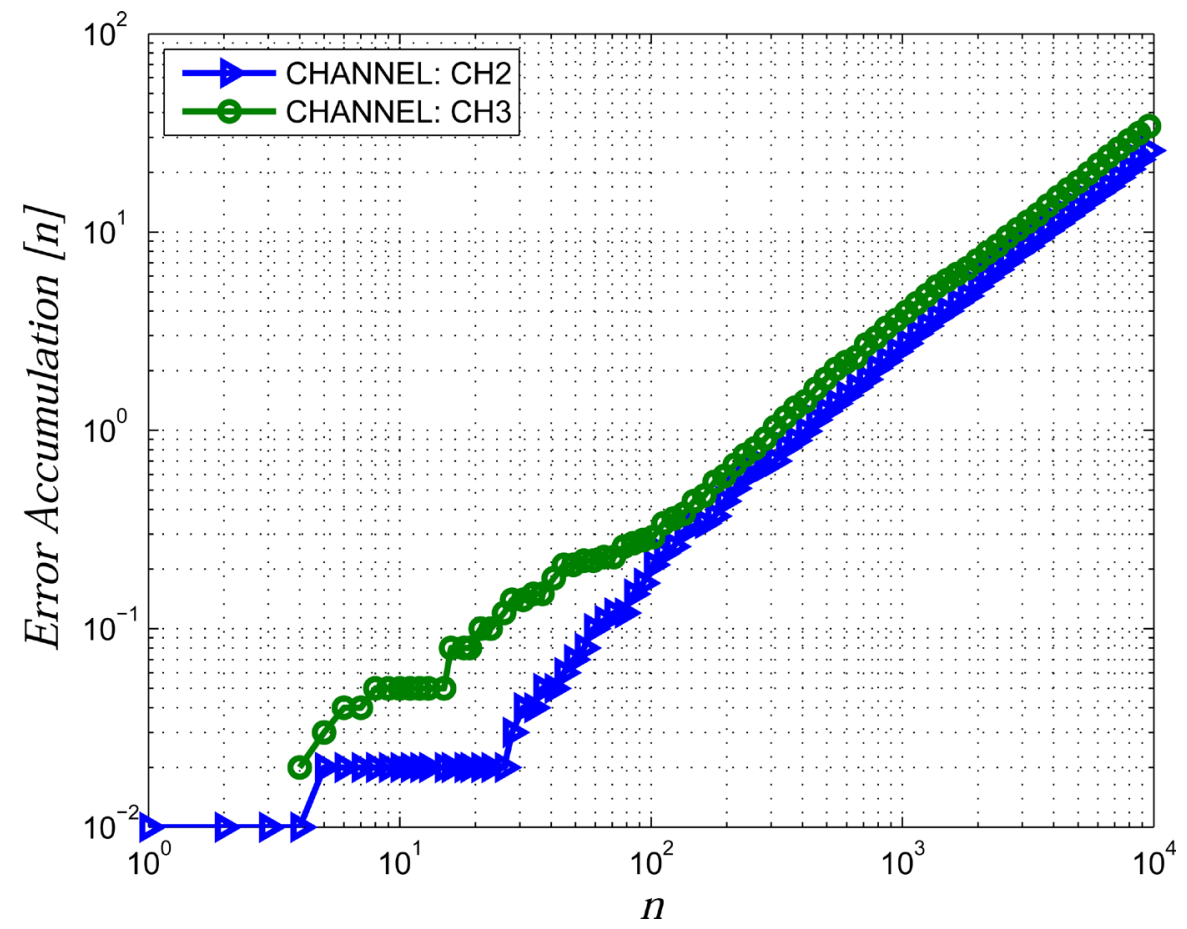

Figure 14. Error Accumulation as a function of iteration number for two channel cases. The averaged results were obtained from 100 Monte Carlo trials. 
2 and $\mu_{G}=5 \times 10^{-5}$ for channel 3) where the equalizer's tap length was set to 13 $(N=13)$ and $\mathrm{SNR}=20[\mathrm{~dB}]$. According to Figure 12 it is very difficult to see for which channel better equalization performance is obtained from the MSE point of view. From Figure 11 the equalization performance from the ISI point of view is very close for the two channel cases (channel 2 and channel 3). However, according to Figure 13 the difference between the equalization performance for the two channels is seen very clearly. In addition the difference in the equalization performance for the two channels is also seen in Figure 14 in the short range while in the long term the difference in the equalization performance resembles the difference in the equalization performance as is seen in Figure 11.

Figures 15-18 show the simulation results for the ISI, MSE, MConE and Accumulated Error (8) respectively for two different channels (channel 2 (CH2) and channel $3(\mathrm{CH} 3)$ ) and for two different step sizes and equalizer's tap length ( $\mu_{G}=4.4 \times 10^{-5}, N=15$ for channel 2 and $\mu_{G}=7 \times 10^{-5}, N=11$ for channel 3) where $\mathrm{SNR}=15[\mathrm{~dB}]$. According to Figure 16 it is very difficult to see for which channel better equalization performance is obtained from the MSE point of view. From Figure 15 the equalization performance from the ISI point of view is very close for the two channel cases (channel 2 and channel 3). However, according to Figure 17 the difference between the equalization performance for the two channels is seen very clearly. In addition the difference in the equalization performance for the two channels is also seen in Figure 18 in the short range while in the long term the difference in the equalization performance resembles the difference in the equalization performance as is seen in Figure 15.

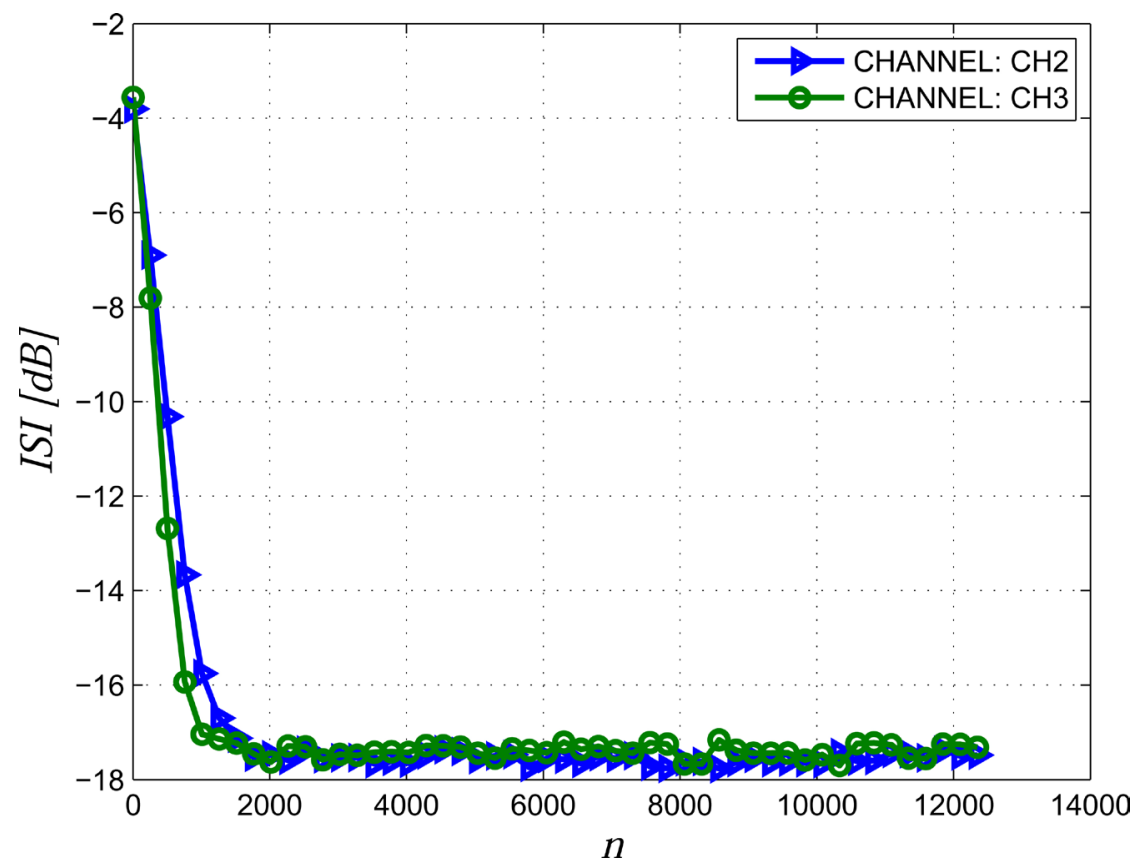

Figure 15. ISI as a function of iteration number for two channel cases. The averaged results were obtained from 100 Monte Carlo trials. 


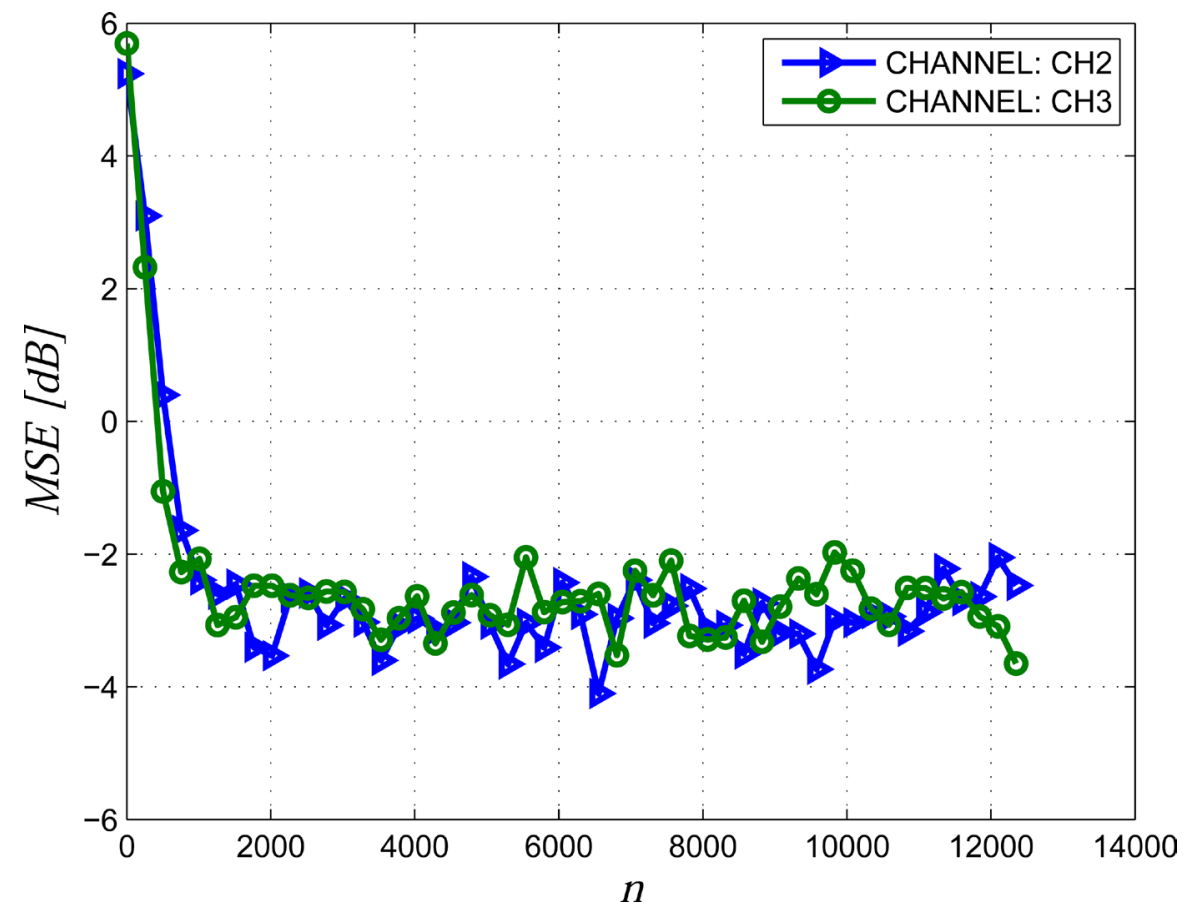

Figure 16. MSE as a function of iteration number for two channel cases. The averaged results were obtained from 100 Monte Carlo trials.

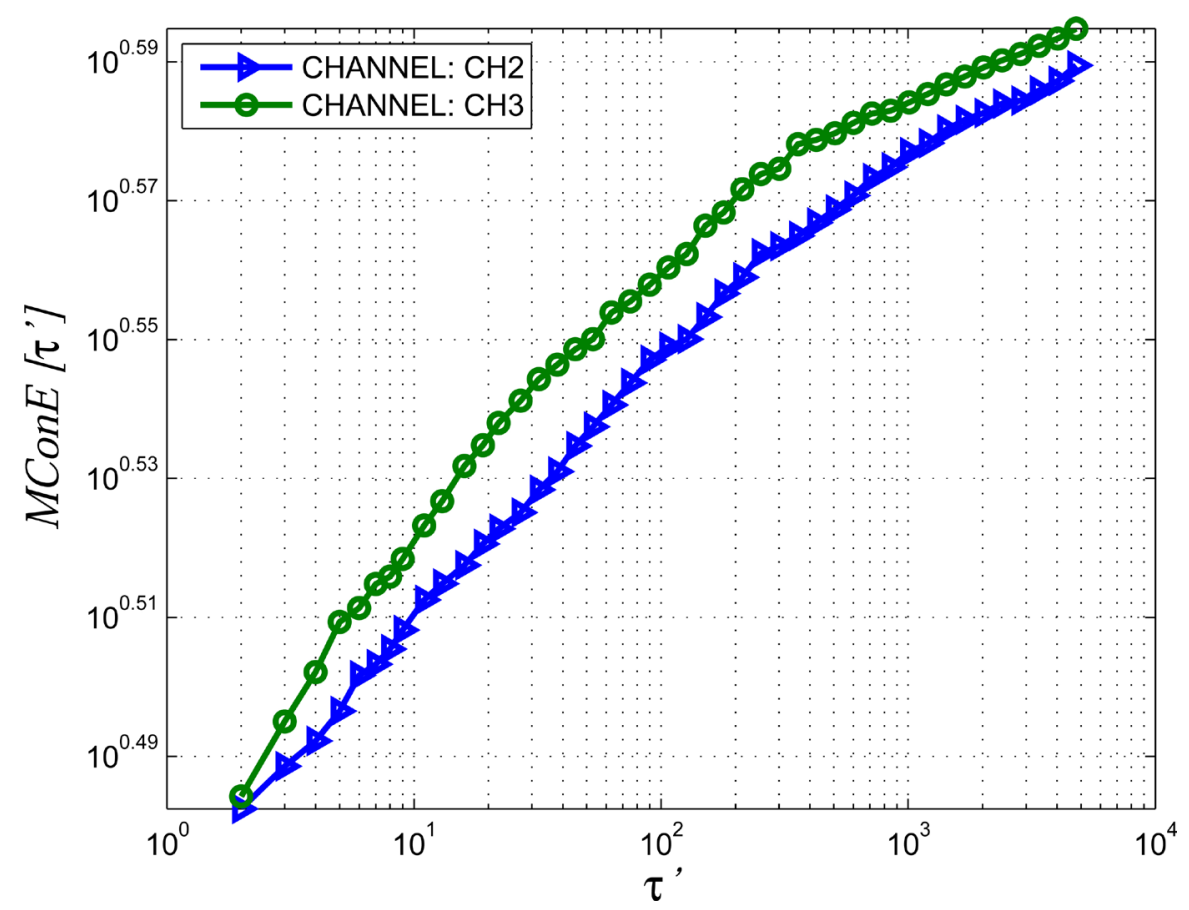

Figure 17. MConE as a function of the window length for two channel cases. The averaged results were obtained from 100 Monte Carlo trials. 


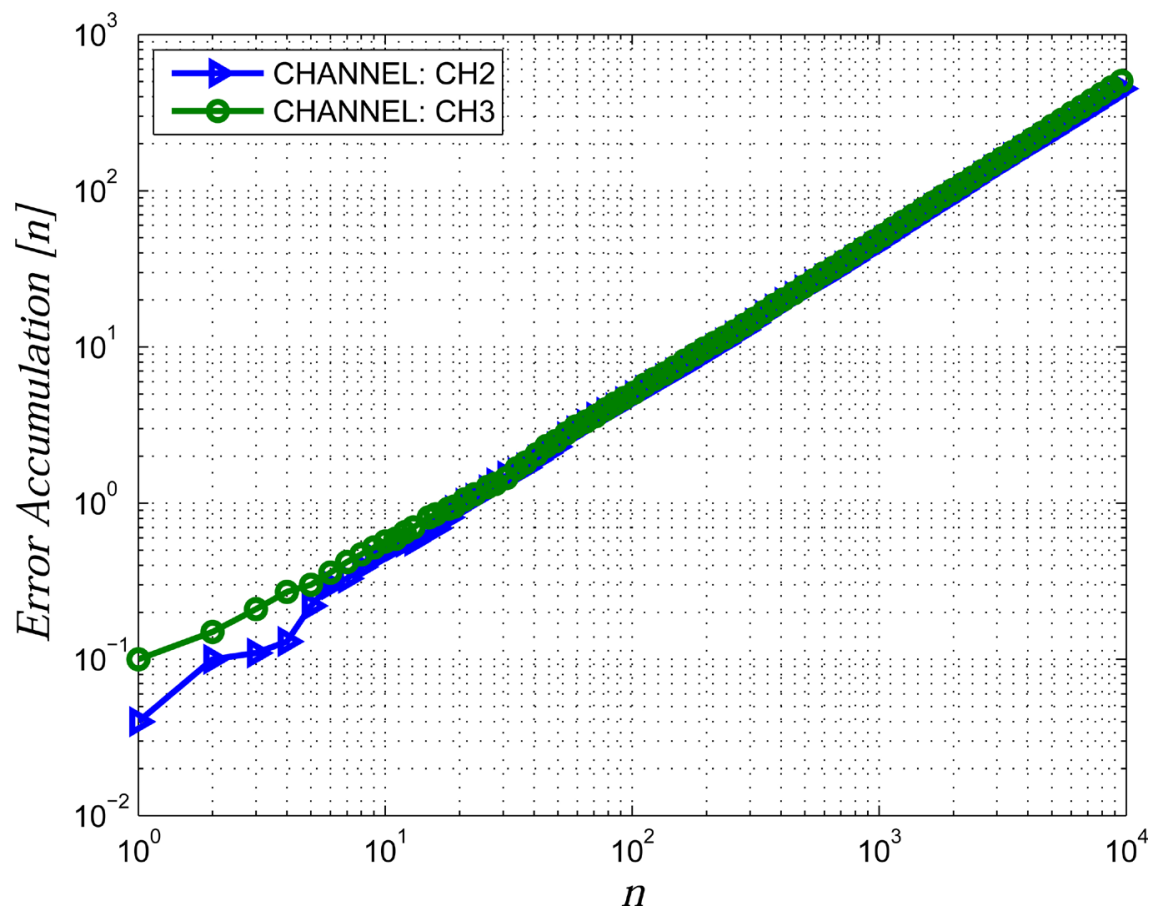

Figure 18. Error Accumulation as a function of iteration number for two channel cases. The averaged results were obtained from 100 Monte Carlo trials.

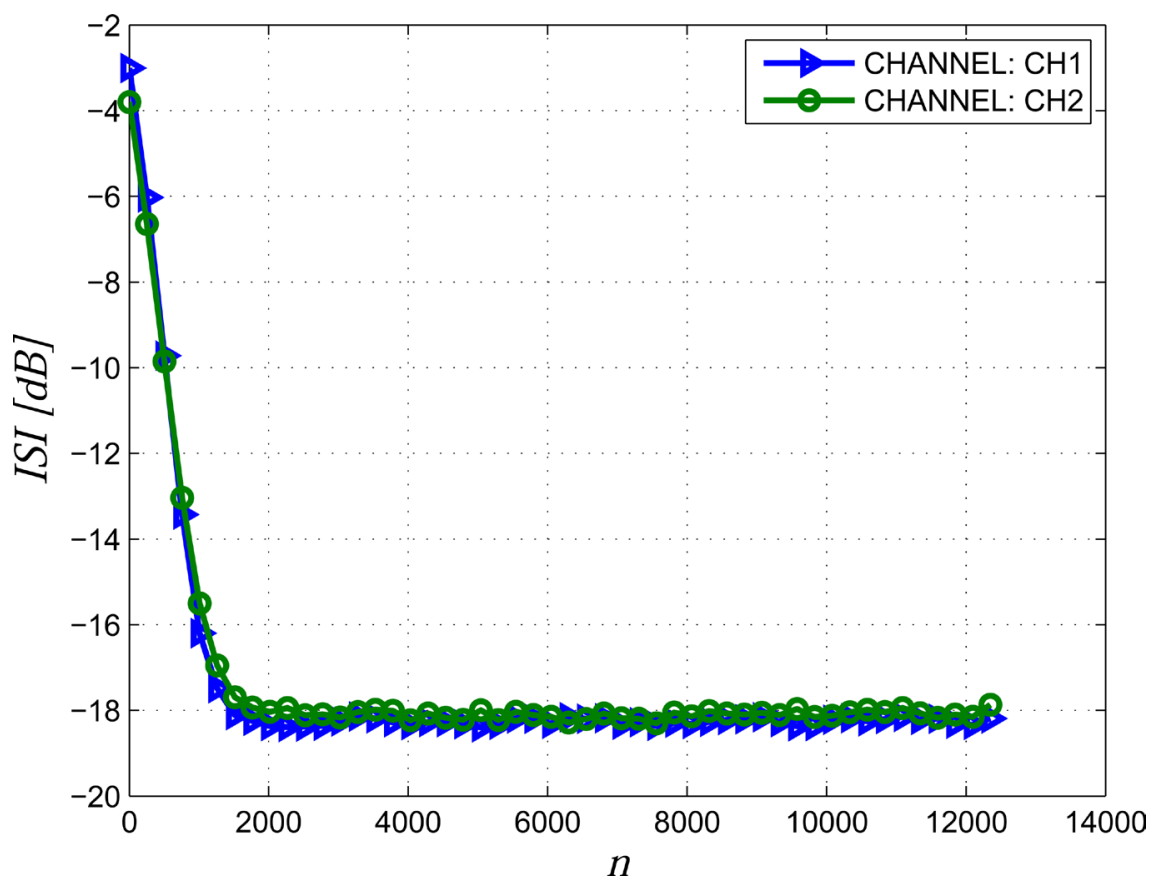

Figure 19. ISI as a function of iteration number for two channel cases. The averaged results were obtained from 100 Monte Carlo trials.

Figures 19-22 show the simulation results for the ISI, MSE, MConE and Accumulated Error (8) respectively for two different channels (channel $1(\mathrm{CH} 1)$ and channel $2(\mathrm{CH} 2))$ and for two different step sizes and equalizer's tap length ( $\mu_{G}=4.5 \times 10^{-5}, N=9$ for channel 1 and $\mu_{G}=4 \times 10^{-5}, N=19$ for channel 2) where $S N R=20[\mathrm{~dB}]$. According to Figure 20 it is very difficult to see for 
which channel better equalization performance is obtained from the MSE point of view. From Figure 19 the equalization performance from the ISI point of view is very close for the two channel cases (channel 1 and channel 2). However, according to Figure 21 the difference between the equalization performance for the two channels is seen very clearly. In addition the difference in the

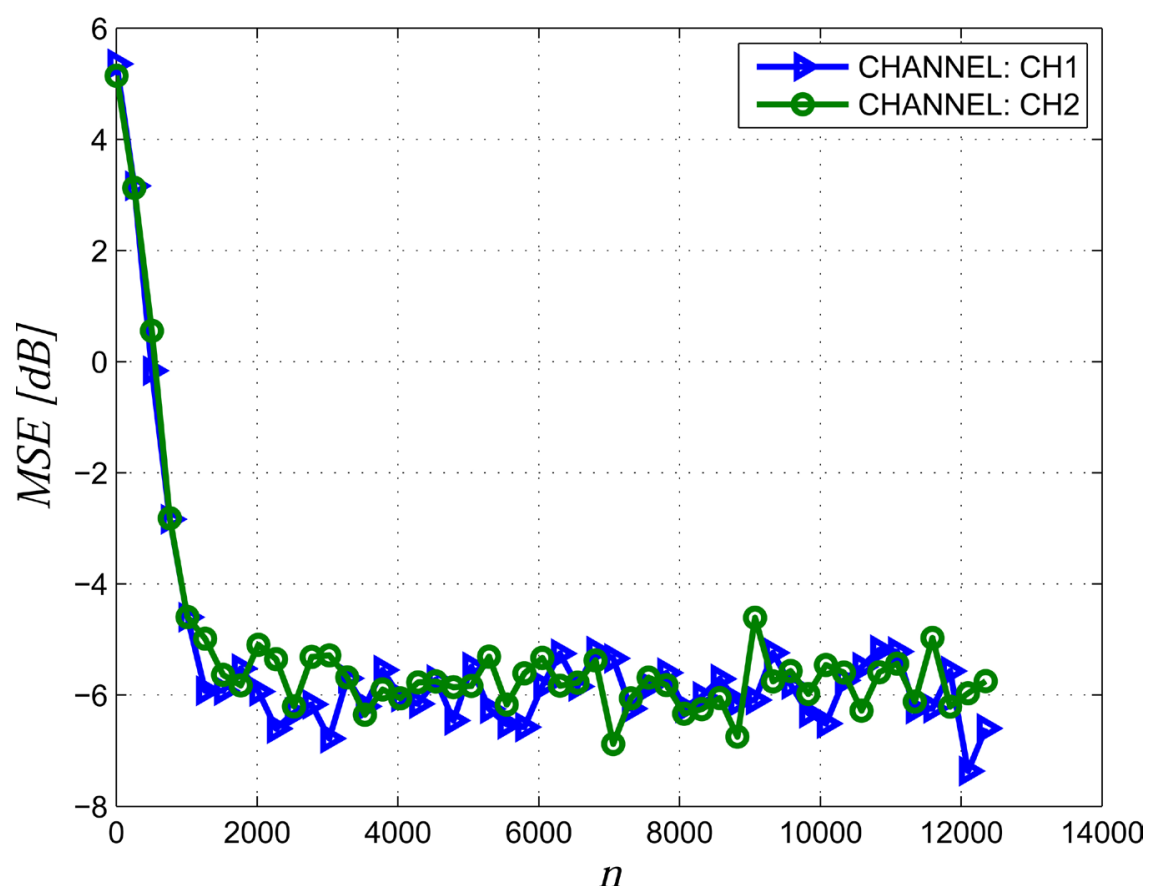

Figure 20. MSE as a function of iteration number for two channel cases. The averaged results were obtained from 100 Monte Carlo trials.

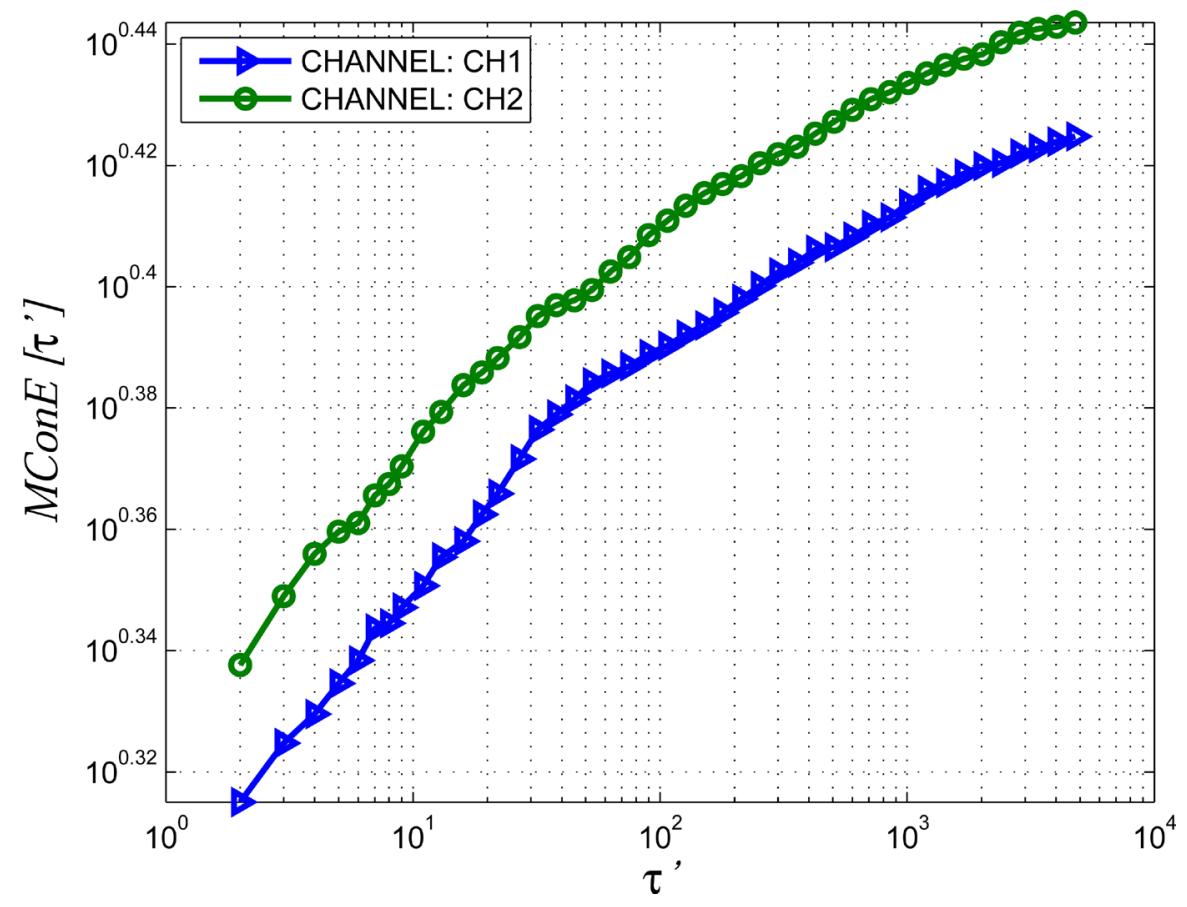

Figure 21. MConE as a function of the window length for two channel cases. The averaged results were obtained from 100 Monte Carlo trials. 


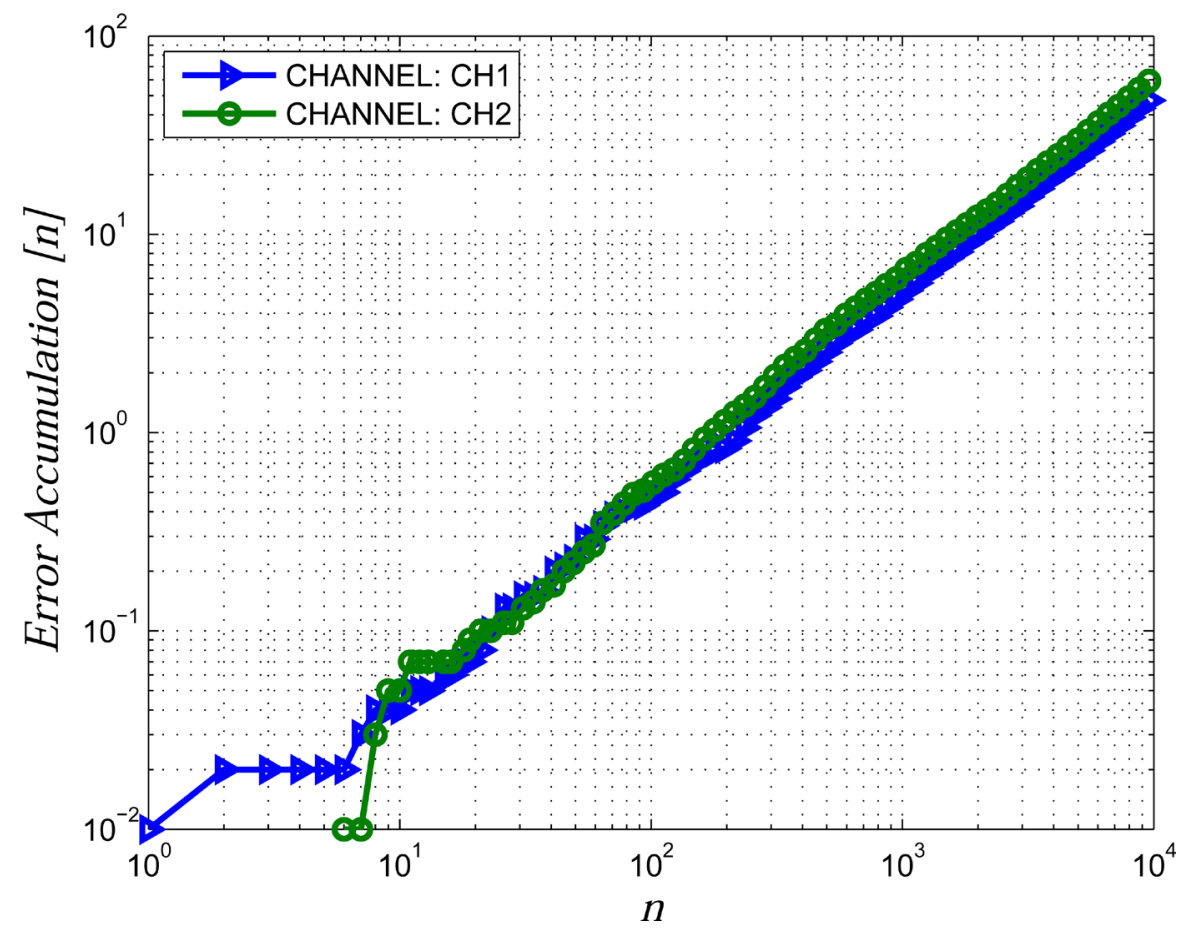

Figure 22. Error Accumulation as a function of iteration number for two channel cases. The averaged results were obtained from 100 Monte Carlo trials.

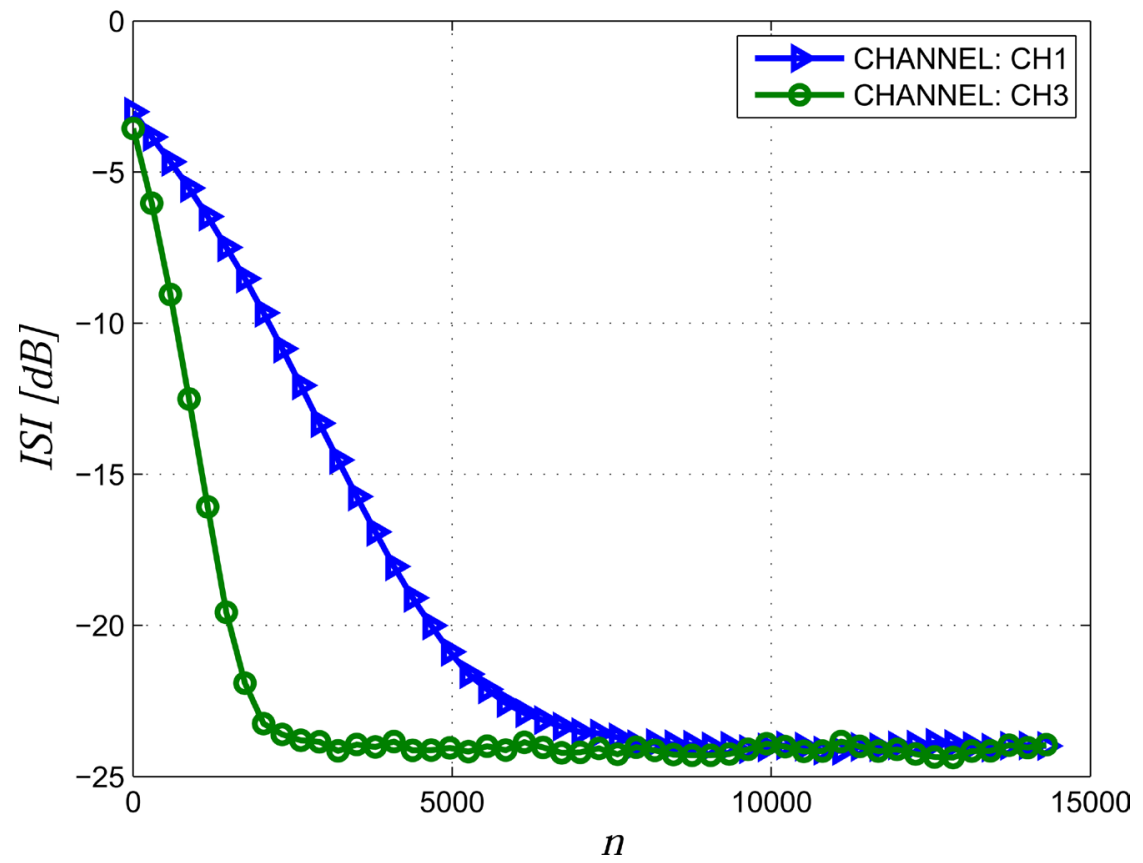

Figure 23. ISI as a function of iteration number for two channel cases. The averaged results were obtained from 100 Monte Carlo trials.

equalization performance for the two channels is also seen in Figure 22 in the short range while in the long term the difference in the equalization performance resembles the difference in the equalization performance as is seen in Figure 19.

Figures 23-26 show the simulation results for the ISI, MSE, MConE and Ac- 
cumulated Error (8) respectively for two different channels (channel 1 (CH1) and channel $3(\mathrm{CH} 3)$ ) and for two different step sizes and equalizer's tap length ( $\mu_{G}=1 \times 10^{-5}, N=13$ for channel 1 and $\mu_{G}=3 \times 10^{-5}, N=7$ for channel 2) where $S N R=20[\mathrm{~dB}]$. According to Figure 24 it is very difficult to see for which channel better equalization performance is obtained from the MSE point of view. From Figure 23 the equalization performance from the ISI point of view is very

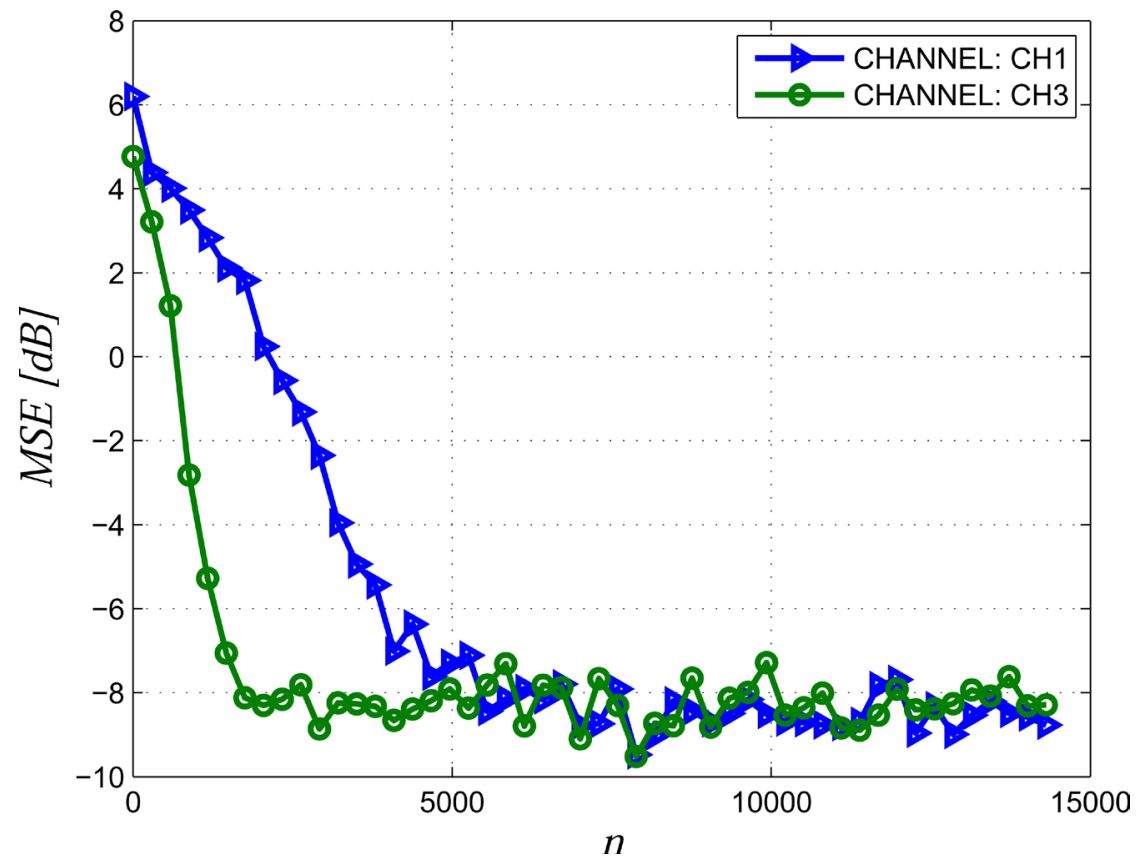

Figure 24. MSE as a function of iteration number for two channel cases. The averaged results were obtained from 100 Monte Carlo trials.

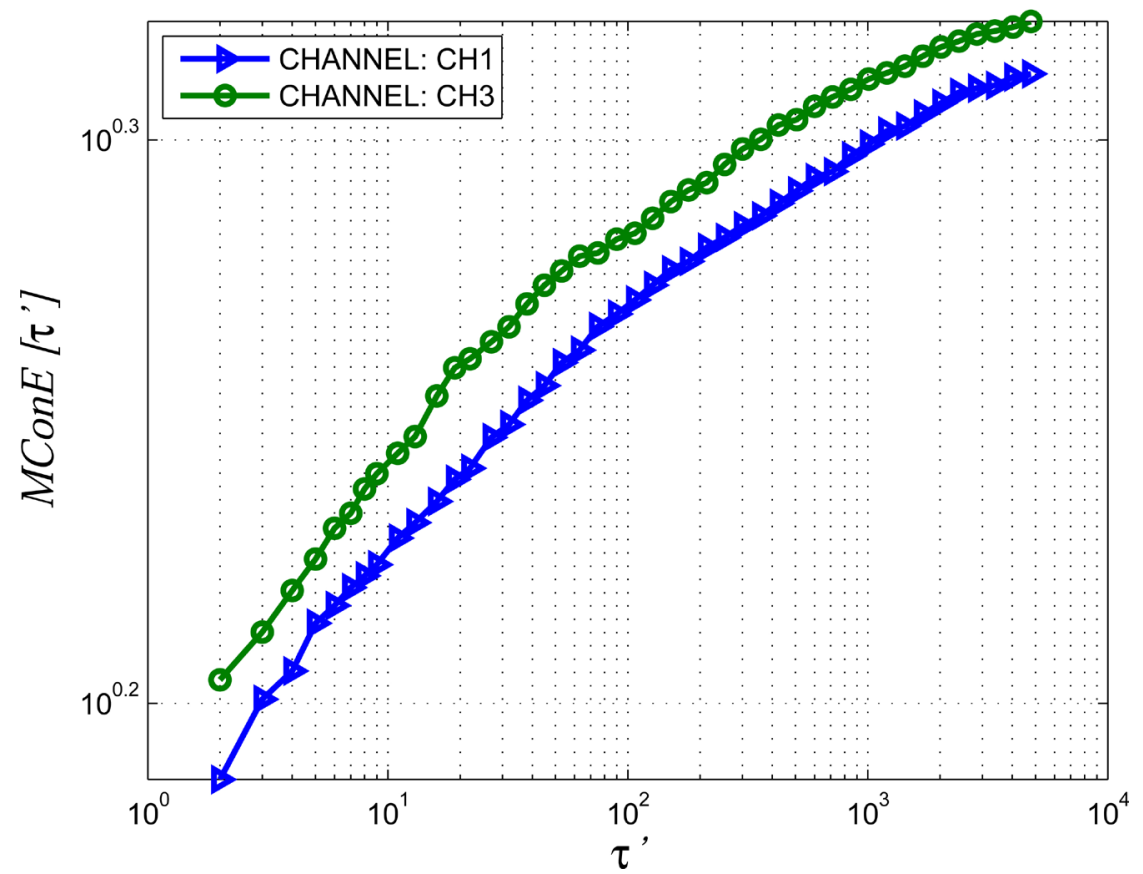

Figure 25. MConE as a function of the window length for two channel cases. The averaged results were obtained from 100 Monte Carlo trials. 


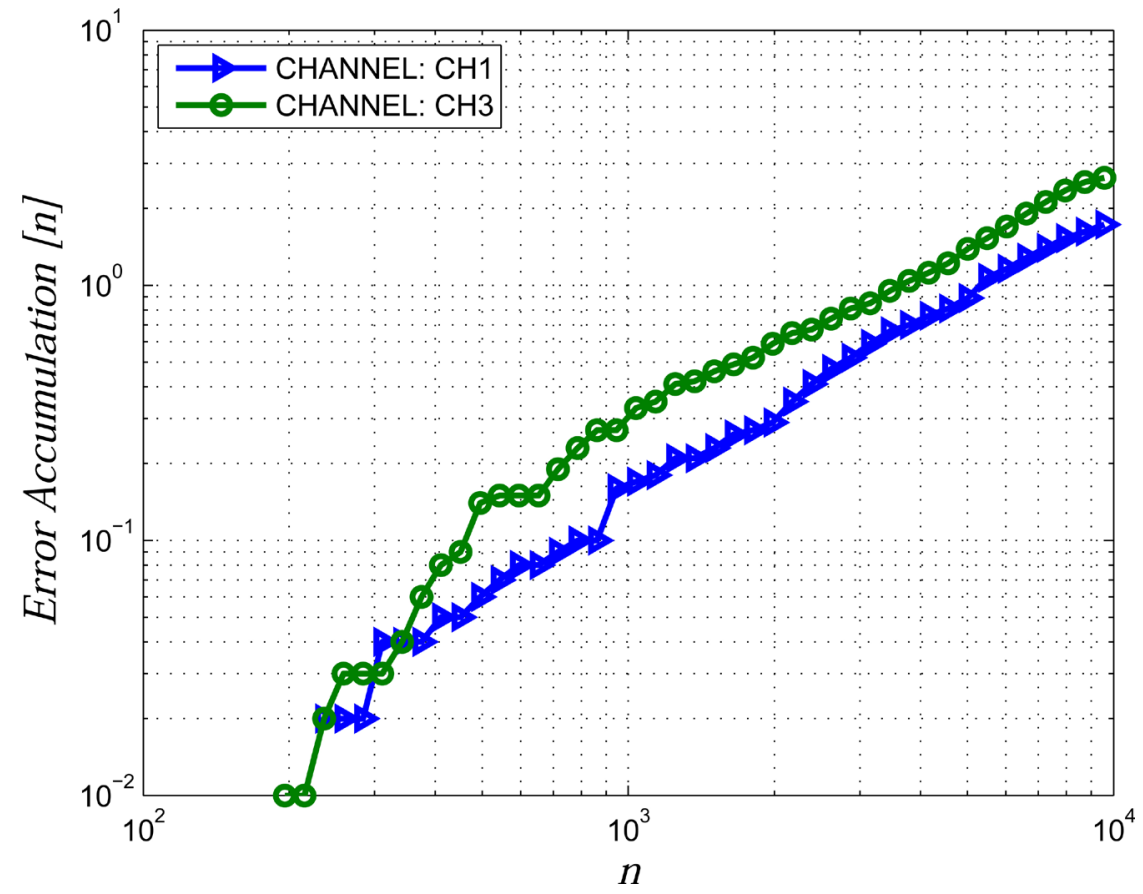

Figure 26. Error Accumulation as a function of iteration number for two channel cases. The averaged results were obtained from 100 Monte Carlo trials.

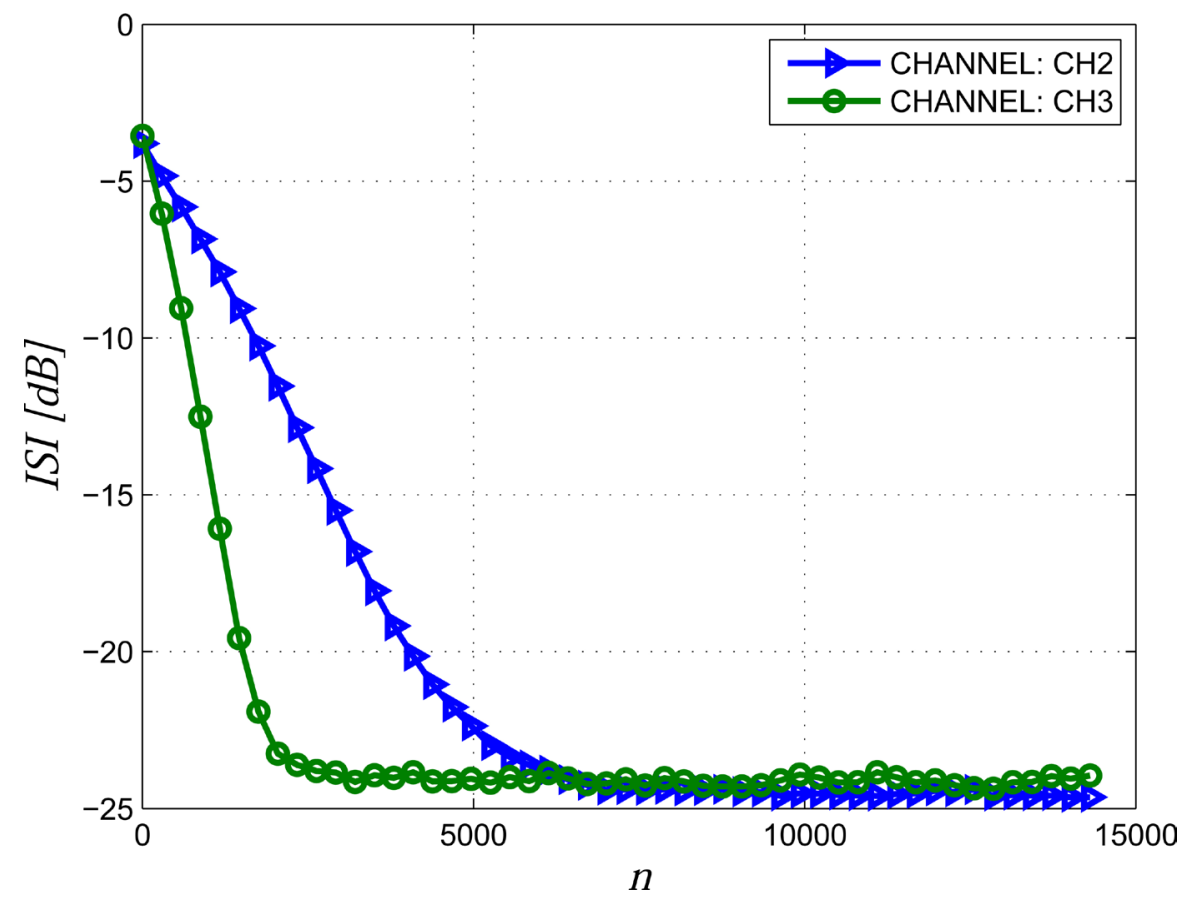

Figure 27. ISI as a function of iteration number for two channel cases. The averaged results were obtained from 100 Monte Carlo trials.

close for the two channel cases (channel 1 and channel 3). However, according to Figure 25 and Figure 26 the difference between the equalization performance for the two channels is seen very clearly.

Figures 27-30 show the simulation results for the ISI, MSE, MConE and Accumulated Error (8) respectively for two different channels (channel $2(\mathrm{CH} 2)$ 
and channel $3(\mathrm{CH} 3))$ and for two different step sizes and equalizer's tap length ( $\mu_{G}=1 \times 10^{-5}, N=13$ for channel 2 and $\mu_{G}=3 \times 10^{-5}, N=7$ for channel 3) where $\mathrm{SNR}=20[\mathrm{~dB}]$. According to Figure 28 it is very difficult to see for which channel better equalization performance is obtained from the MSE point of view. From Figure 27 the equalization performance from the ISI point of view is very close for the two channel cases (channel 2 and channel 3). However, according

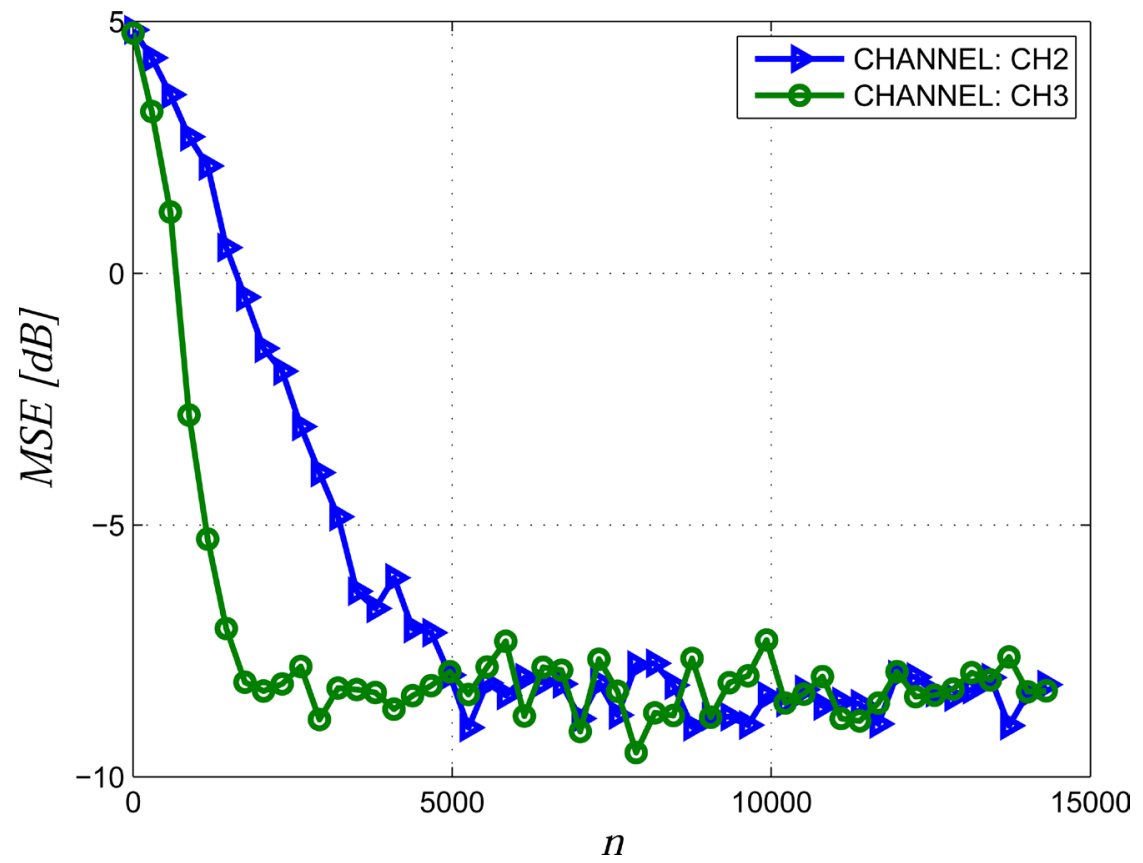

Figure 28. MSE as a function of iteration number for two channel cases. The averaged results were obtained from 100 Monte Carlo trials.

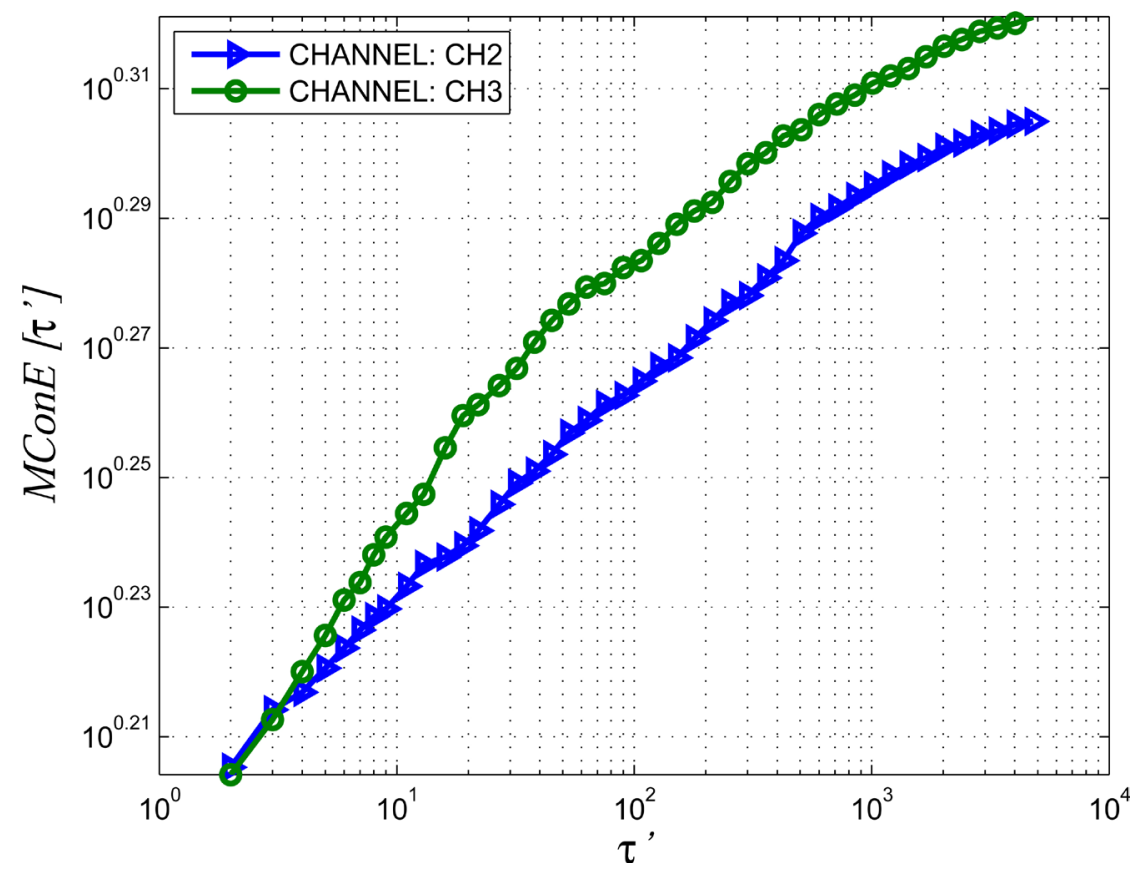

Figure 29. MConE as a function of the window length for two channel cases. The averaged results were obtained from 100 Monte Carlo trials. 


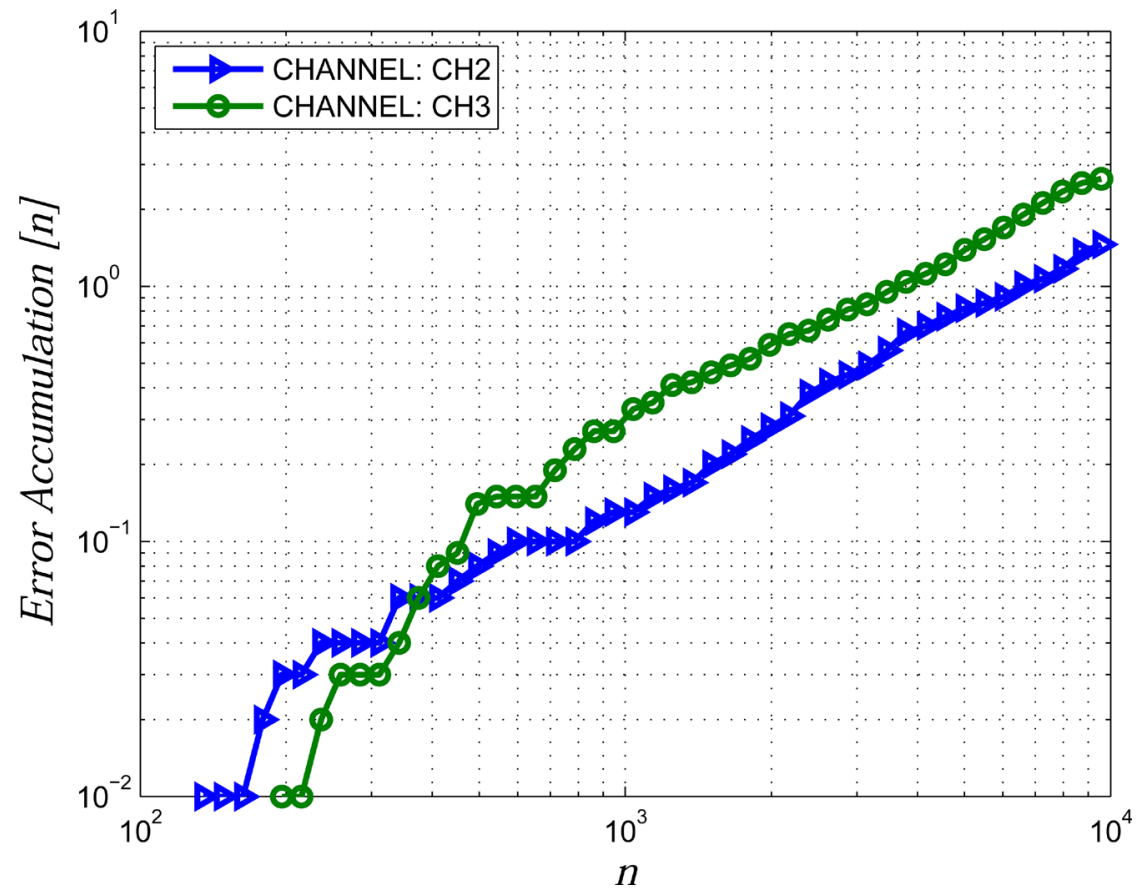

Figure 30. Error Accumulation as a function of iteration number for two channel cases. The averaged results were obtained from 100 Monte Carlo trials.

to Figure 29 and Figure 30 the difference between the equalization performance for the two channels is seen very clearly.

\section{Conclusion}

In this paper, we proposed a new tool for analyzing the equalization performance in the convergence state which can be considered as an additional tool to the literature known methods (ISI, MSE, BER). The new proposed tool is based on the MTIE criterion that is used for the specification of clock stability requirements in telecommunications standards. This new tool preserves the short term statistical information unlike the BER, ISI and MSE method. Thus, our new proposed tool can supply us short term as well as long term statistical information. Simulation results have shown that with our new proposed tool, difference in the equalization performance comparison was clearly seen in the convergence state while this was not the case with the MSE and ISI method. Thus, our new proposed tool for analyzing the equalization performance in the convergence state might be considered as a more sensitive tool compared to the ISI and MSE method.

\section{Acknowledgments}

We thank the Editor and the referee for their comments.

\section{References}

[1] Johnson, R., Schniter, P., Endres, T.J., Behm, J.D., Brown, D.R., Casas, R.A., et al. (1998) Blind Equalization Using the Constant Modulus Criterion: A Review. Pro- 
ceedings of the IEEE, 86, 1927-1950. https://doi.org/10.1109/5.720246

[2] Weerackody, V. and Kassam, S.A. (1991) Variable Step-Size Blind Adaptive Equalization Algorithms. IEEE International Symposium on Circuits and Systems, 1, 718721.

[3] Proakis, J.G. (2001) Digital Communications. In: McGraw-Hill Series in Electrical and Computer Engineering. Communications and Signal Processing, McGraw-Hill.

[4] Nikias, C.L and Mendel, J.M. (1993) Signal Processing with Higher-Order Spectra. IEEE Signal Processing Magazine, 10, 10-37. https://doi.org/10.1109/79.221324

[5] Silva, M.T.M. and Arenas-Garcia, J. (2013) A Soft-Switching Blind Equalization Scheme via Convex Combination of Adaptive Filters. IEEE Transactions on Signal Processing, 61, 1171-1182. https://doi.org/10.1109/TSP.2012.2236835

[6] Godard, D. (1980) Self-Recovering Equalization and Carrier Tracking in Two-Dimensional Data Communication Systems. IEEE Transactions on Communications, 28, 1867-1875. https://doi.org/10.1109/TCOM.1980.1094608

[7] Treichler, J. and Agee, B. (1983) A New Approach to Multipath Correction of Constant Modulus Signals. IEEE Transactions on Acoustics, Speech, and Signal Processing, 31, 459-472. https://doi.org/10.1109/TASSP.1983.1164062

[8] Wesolowski, K. (1992) Analysis and Properties of the Modified Constant Modulus Algorithm for Blind Equalization. European Transactions on Telecommunications, 3, 225-230. https://doi.org/10.1002/ett.4460030303

[9] Oh, K.N. and Chin, Y.O. (1995) Modified Constant Modulus Algorithm: Blind Equalization and Carrier Phase Recovery Algorithm. 1995 IEEE International Conference on Communications, 1, 498-502.

[10] Yang, J., Werner, J.-J. and Dumont, G.A. (2002) The Multimodulus Blind Equalization and Its Generalized Algorithms. IEEE Journal on Selected Areas in Communications, 20, 997-1015. https://doi.org/10.1109/JSAC.2002.1007381

[11] Lindsey, W.C., Ghazvinian, F., Hagmann, W.C. and Dessouky, K. (1985) Network Synchronization. Proceedings of the IEEE, 73, 1445-1467.

https://doi.org/10.1109/PROC.1985.13317

[12] Kartaschoff, P. (1991) Synchronization in Digital Communications Networks. Proceedings of the IEEE, 79, 1019-1028. https://doi.org/10.1109/5.84979

[13] Bellamy, J.C. (1995) Digital Network Synchronization. IEEE Communications Magazine, 33, 70-83. https://doi.org/10.1109/35.372197

[14] Bregni, S. and Maccabruni, S. (2000) Fast Computation of Maximum Time Interval Error by Binary Decomposition. IEEE Transactions on Instrumentation and Measurement, 49, 1240-1244. https://doi.org/10.1109/19.893262

[15] (1996) Definitions and Terminology for Synchronization Networks. ITU-T, Geneva, Switzerland.

[16] (1997) Timing Characteristics of Primary Reference Clocks. ITU-T, Geneva, Switzerland.

[17] (1998) Timing Requirements of Slave Clocks Suitable for Use as Node Clocks in Synchronization Networks. ITU-T, Geneva, Switzerland.

[18] (1996) Timing Characteristics of SDH Equipment Slave Clocks (SEC). ITU-T, Geneva, Switzerland.

[19] EN ETSI. 300462 Transmission and Multiplexing (TM); Generic Requirements for Synchronization Networks.

[20] Bregni, S. (1996) Measurement of Maximum Time Interval Error for Telecommunications Clock Stability Characterization. IEEE Transactions on Instrumentation 
and Measurement, 45, 900-906. https://doi.org/10.1109/19.536708

[21] Nikias, C.L. and Petropulu, A.P. (1993) Higher-Order Spectra Analysis: A Nonlinear Signal Processing Framework. Prentice Hall, Upper Saddle River.

[22] Pinchas, M. (2012) The Whole Story behind Blind Adaptive Equalizers/Blind Deconvolution. Bentham Science Publishers, Sharjah.

[23] Li, Y. and Ding, Z. (1996) Global Convergence of Fractionally Spaced Godard (CMA) Adaptive Equalizers. IEEE Transactions on Signal Processing, 44, 818-826. https://doi.org/10.1109/78.492535

[24] Sharma, V. and Raj, V.N. (2005) Convergence and Performance Analysis of Godard Family and Multimodulus Algorithms for Blind Equalization. IEEE Transactions on Signal Processing, 53, 1520-1533. https://doi.org/10.1109/TSP.2005.843725

[25] Diniz, P.S.R. (1997) Adaptive Filtering. Springer, USA. https://doi.org/10.1007/978-1-4419-8660-3

[26] Chi, C.-Y., Feng, C.-C., Chen, C.-H. and Chen, C.-Y. (2006) Blind Equalization and System Identification: Batch Processing Algorithms, Performance and Applications. Springer Science \& Business Media, New York.

[27] Khan, M.L.R., Wondimagegnehu, M.H. and Shimamura, T. (2009) Blind Channel Equalization with Amplitude Banded Godard and Sato Algorithms. Journal of Communications, 4, 388-395.

[28] Ding, Z. (2000) Adaptive Filters for Blind Equalization. In: Madisetti, V.K. and Williams, D., Eds., The Digital Signal Processing Handbook, Chapter 24. CRC Press, Boca Raton, 1-19. https://doi.org/10.1201/9781420046076-c24

[29] Dobrogowski, A. and Kasznia, M. (2001) Time Effective Methods of Calculation of Maximum Time Interval Error. IEEE Transactions on Instrumentation and Measurement, 50, 732-741. https://doi.org/10.1109/19.930447

[30] Oscilloquartz SA 4520 GPS-SP.

http://syncarchitect.com/downloads/PDF_0012_4520.pdf

[31] Fiori, S. (2001) A Contribution to (Neuromorphic) Blind Deconvolution by Flexible Approximated Bayesian Estimation. Signal Processing, 81, 2131-2153.

[32] Shalvi, O. and Weinstein, E. (1990) New Criteria for Blind Deconvolution of Nonminimum Phase Systems (Channels). IEEE Transactions on Information Theory, 36, 312-321. https://doi.org/10.1109/18.52478 


\section{Abbreviations}

MSE- Mean Square Error

ISI- Intersymbol Interference

BER- Bit Error Rate

MTIE- Maximum Time Interval Error

FIR- Finite Impulse Response

QAM- Quadrature Amplitude Modulation

TE- Time Error

TIE- Time Interval Error

SNR- Signal to Noise Ratio

ConE- Convolution Error

MConE- Maximum Convolution Error

E A- Error Accumulation

Submit or recommend next manuscript to SCIRP and we will provide best service for you:

Accepting pre-submission inquiries through Email, Facebook, LinkedIn, Twitter, etc. A wide selection of journals (inclusive of 9 subjects, more than 200 journals)

Providing 24-hour high-quality service

User-friendly online submission system

Fair and swift peer-review system

Efficient typesetting and proofreading procedure

Display of the result of downloads and visits, as well as the number of cited articles

Maximum dissemination of your research work

Submit your manuscript at: http://papersubmission.scirp.org/

Or contact jsip@scirp.org 